\title{
An electromechanical moving load fixed node position and fixed node number railway power supply systems optimization model
}

\author{
Lars Abrahamsson ${ }^{\mathrm{a}, *}$, Stefan Östlund ${ }^{\mathrm{b}}$, Thorsten Schütte ${ }^{\mathrm{c}}$, Lennart Söder ${ }^{\mathrm{a}}$ \\ ${ }^{a}$ School of Electrical Engineering, Electric Power Systems, KTH Royal Institute of \\ Technology, Teknikringen 33, SE-100 44 Stockholm, Sweden \\ ${ }^{b}$ School of Electrical Engineering, Electrical Energy Conversion, KTH Royal Institute of \\ Technology, Teknikringen 33, SE-100 44 Stockholm, Sweden \\ ${ }^{c}$ Atkins Sverige AB, Kopparbergsvägen 8, SE-722 13 Västerås, Sweden
}

\begin{abstract}
This paper presents an optimization model for simulations of railway power supply systems. It includes detailed power systems modeling, train movements in discretized time considering running resistance and other mechanical constraints, and the voltage-drop-induced reduction of possible train tractive forces. The model has a fixed number of stationary power system nodes, which alleviates optimized operation over time. The proposed model uses SOS2 (Special Ordered Sets of type 2) variables to distribute the train loads to the two most adjacent power system nodes available.

The impacts of the number of power system nodes along the contact line and the discretized time step length on model accuracy and computation times are investigated.

The program is implemented in GAMS. Experiences from various solver choices are also discussed. The train traveling times are minimized in the example. Other studies could e.g. consider energy consumption minimization. The numerical example is representative for a Swedish decentralized, rotary-converter fed railway power supply system. The proposed concept

\footnotetext{
*Corresponding author: Telephone +46 879070 58, Mobile +467025622 82, Fax +4687906510

Email addresses: lars.abrahamsson@ee.kth.se (Lars Abrahamsson), stefan.ostlund@ee.kth.se (Stefan Östlund), thorsten.schutte@atkinsglobal.com (Thorsten Schütte), lennart.soder@ee.kth.se (Lennart Söder)
} 
is however generalizable and could be applied for all kinds of moving load power system studies.

Keywords: optimization, railway, power systems, special ordered sets of type 2 (SOS2), MINLP, moving loads

\section{Introduction}

This paper is a further developed model of the preliminary results presented in (Abrahamsson and Söder, 2012), moreover, in this paper the models used are presented completely and exhaustively.

The paper shows in various numerical examples that the model produces reliable results. The computational times and details in accuracy of results are compared for various number of power system nodes along the contact line and various discretized time step lengths.

This paper presents and proposes an optimization model for simulations of railway power supply systems, including train movements in discretized time considering running resistance and other mechanical constraints, as well as the voltage-drop-induced reduction of possible train tractive forces.

The proposed model uses SOS2 variables to distribute the train loads to the two most adjacent power system nodes available. The program is implemented in GAMS (GAMS, 2008). Experiences from various solver (GAMS, 2011) choices are also presented. The train traveling times are minimized in the example.

The numerical examples are representative for Swedish decentralized, rotary-converter fed railway power supply systems with contact lines using Booster Transformer (BT) (Hill, 1994) technology. The proposed concept is however generalizable and could with some modifications be applied for all kinds of moving load power system studies.

\subsection{Background and Motivation}

There are some well-known problems associated with power system modeling of moving electrical loads for load flow studies. One of the first to ventilate these was (Talukdar and Koo, 1977). In (Talukdar and Koo, 1977) also the main differences between public power systems and railway power supply systems are extensively discussed and explained. Typically, the loads 
are neither fixed power, fixed current, or even fixed location, they vary heavily.

If each moving load is represented by an electric node in the power system, the ordering and numbering of nodes and loads (in this case train vehicles) will soon need detailed bookkeeping algorithms (Abrahamsson, 2008; Arboleya et al., 2012). Such bookkeeping will involve: train numbers, train locations, node numbers, which trains are in traffic or not, which nodes coincide and should be merged or not, etc.

In order to keep the admittance matrix element values within reasonable limits, electrical nodes cannot be located too close to one another. If two trains are very close to each other, or if a train is right under a catenary junction or substation of any kind, the admittances might tend to infinity. This can be overcome with an additional bookkeeping, that merges nodes if they come too electrically close to each other (Abrahamsson, 2008; Talukdar and Koo, 1977). Another option is to introduce minimally allowed impedance values (Arboleya et al., 2012). This bookkeeping obstructs a straight-forward modeling. It should however be noted, that from a strict computational point-of-view, the bookkeeping and updating of matrices between each time step is not a severe problem (Talukdar and Koo, 1977).

Besides the bookkeeping issues for merging nodes, the number of nodes in the power system varies also when trains start or stop and run in and out of the studied part of the system. If the number of nodes change between a pair of consecutive time steps, it is untrivial to optimize train operation or power system operation over time. That is because optimization problems cannot change in size during computation, and migrating the book-keeping algorithms into an optimization program without introducing a multitude of binary variables is not doable. Such a model cannot be rejected without investigation, but is however out of the scope of this paper.

The proposed solution, c.f. Section 3, use a fixed number of stationary nodes to represent the RPSS electrically. This alleviates temporal optimization. The solution makes use of SOS2 variables in a way that the above described bookkeeping becomes unnecessary and these issues are now treated internally in the optimization model.

Another solution to the bookkeeping problem has been proposed in $\mathrm{Ar}-$ boleya et al., 2012), using incidence matrices and graphs. Drawbacks with that proposal are that trains never leave the power system, trains not connected to the system are instead assigned extremely high numerical values on impedances. This results in an undesired wide spread range of impedance 
magnitudes (Talukdar and Koo, 1977), and might cause computational problems in studies where there are many trains not in service for the moment. The sizes and locations of the loads are in (Arboleya et al., 2012) determined in an external program, and therefore the load flows are (and have to be) solved separately for each time step.

The idea to shop up the catenary in discrete segments in order to create a power system with a fixed number of stationary nodes was first introduced in (Talukdar and Koo, 1977). That model moved the electrical load of each train from its actual position to the most adjacent node.

\subsection{The Idea Behind the Proposed Model}

In this paper, the solution by (Talukdar and Koo, 1977) has been refined. The discretization errors are reduced by a linear weighting between the two most adjacent nodes instead of using just the most adjacent node. Moreover, no formalized bookkeeping of the relations between trains, their positions, and the power system are needed, because the linear weighting has been seamed within the model. This altogether creates a model that is comparatively easy to understand for developers and users, and that also manages optimization over time.

This is made possible by the use of SOS2 (Special Ordered Sets of type 2) variables, by which the trains exact position can be linearly approximated in the power system without losing the exact train position for the mechanical part of the model. The obtained linear approximative weighting between the two electrical nodes most adjacent to the train is used also for train power consumption and train voltages.

In many Railway Power Supply System (RPSS) simulator models the power system operation of each discretized snapshot in time acts as if there where no future and no past. In contrast to that, the model in this paper is able to optimize electrical and mechanical RPSS operation also temporally. What is optimal if things would stay the same forever is normally not optimal when the modeling includes the changes of the system.

Optimization in the time dimension will typically be useful for studies of on-vehicle (Iannuzzi and Tricoli, 2012; Ciccarelli et al., 2012; Barrero et al., 2008) and railside (Barrero et al., 2008; Iannuzzi et al., 2011, 2012) energy storage.

Present-day models (Shiokawa and Tagaki, 2012; López-López et al., 2012; Ciccarelli et al. 2012) often consider catenary voltage levels and train speeds for determining the optimal operation of energy storage equipment. 
In temporal studies one can also consider the fact that the limit for shorthold overloading of equipment normally significantly exceeds the long-term overloading of equipment.

\subsection{SOS2}

SOS2 variables are, in vector form, nonzero for at most two adjacent elements in the vector. The concept can be generalized for higher dimensions. SOS2 is traditionally used for sampling nonlinear functions into piecewise linear ones. The application of SOS2 presented in this paper is as far as the authors know completely new and unpublished, besides the presentation of the concept and the preliminary results in (Abrahamsson and Söder, 2012).

How the SOS2 variables work in practice in this particular problem is illustrated graphically in Section 4.2 .

Theoretically, an SOS2 variable $\alpha_{i}$,

$$
c_{1, i} \leq \alpha_{i} \leq c_{2, i}
$$

where $c_{1, i}$ and $c_{2, i}$ are the upper and lower bounds of that variable, can be described mathematically equivalent by a real variable $b_{i}$ and the binary variables $\beta_{i}$ and $\beth_{i}$ in the following,

$$
\begin{aligned}
b_{i} & \leq \beta_{i} \cdot c_{2} \\
b_{i} & \geq \beta_{i} \cdot c_{1} \\
\sum_{i} \beta_{i} & \leq 2 \\
\beta_{i}+\beta_{i+1} & \geq \beth_{i} \\
\sum_{i} \beth_{i} & =1 .
\end{aligned}
$$

It should however be noted that SOS2 variables algorithmically are treated more efficient in some solvers than the above mathematical equivalent. In (2) and (3), the lower and upper bounds of $b_{i}$ are defined. For an SOS2 variable it is said implicitly, but here it is stated explicitly that it can only be nonzero under some conditions. Those conditions are defined in (4) where it is stated that $b_{i}$ can be nonzero only on two elements. Equations (5) and (6) together says that these two elements have to be neighbors. 


\section{Terminology Used}

\subsection{Contact Lines}

The contact line, delivering electricity to the trains, is often denoted the catenary.

Physically however, the catenary is just the part of the overhead contact line system which is shaped very much like a catenary. The catenary shape is the curve theoretically assumed by a perfectly flexible and inextensible cord of uniform density and cross section hanging freely from two fixed points.

For convenience, in this paper, as in many other publications, the terms catenary and (overhead) contact line are used synonymously.

When contact lines are not installed as overhead lines, they are normally installed as a third rail alongside the railway tracks. This solution is common in subways.

The pantograph is the current collector, i.e. the device that connects the train electrically to the catenary.

\subsection{Forces and Efforts, Curves and Diagrams}

In the railway society many people use the term tractive force, whereas others use the term tractive effort for the resulting total motoring mechanical force at the locomotives' wheels. In this paper however, the term tractive force is being used.

Additionally, the graphical representation of the maximal possible tractive force of the train as a function of train velocity and pantograph voltage level, c.f. Figure 1, is sometimes called tractive force curve, sometimes tractive effort curve, sometimes tractive force diagram, and sometimes tractive effort diagram. In this paper however, the term tractive force curve is consequently used.

\section{The Model}

Section 3 starts by presenting the mathematical model used in detail in Section 3.1, whereas the equations and modeling choices are expressed and explained in words in Section 3.2 . 
Table 1: Index Sets

\begin{tabular}{cl}
\hline Set & Description \\
\hline$n, n_{2} \in\{1,2, \ldots, N\}$ & Electrical nodes \\
$g(n) \in\left\{G_{1}, G_{2}\right\}$ & Converter station nodes \\
$c(n) \in\{1,2, \ldots, C\}$ & Catenary nodes \\
$n_{n g}(n) \in\{1,2, \ldots, N\} \backslash\left\{G_{1}, G_{2}\right\}$ & Nodes with no converter \\
$n_{n c}(n) \in\{C+1, C+2, \ldots, N\}$ & Nodes not representing the catenary \\
$t \in\{1,2, \ldots, T\}$ & Train numbers \\
$\delta, \delta \in\{1,2, \ldots, \Delta\}$ & Time steps \\
\hline
\end{tabular}

\subsection{Mathematical Modeling}

The model presented here is constrained to one doubly-fed catenary section. The optimization problem consists of the index sets listed in Table 1, the parameters listed in Table 2, and the variables listed in Table 3,

In a simple BT system with only one catenary section fed from each end-point by converter stations, like the one studied here,

$$
\begin{aligned}
N & =C \\
G_{1} & =1 \\
G_{2} & =N,
\end{aligned}
$$

which makes the index set $n_{n c}$ in Table 1 empty for this study. The concept can however be generalized. The number of time-steps $\Delta$ are calculated such that even severely delayed trains will make it to their destinations before time is up. The model is simplified such that the adhesive train mass is assumed to be the entire mass of the train. This is done to simplify the equations describing braking, coasting, and motoring. Therefore, also rotational inertia in the wheel sets and in the locomotive are disregarded. Slippage is also neglected since the adhesive force between wheel and rail is assumed to always be large enough to use the entire motoring force as tractive force. The model is simplified in this way because the focus of this paper is to promote the idea of fixed-node grid-models for moving load power systems - not to have exact mechanical models of the train-rail interaction.

Moreover, train running resistance due to curves and gradients of the track are not implemented in this model. The track topography is thus 
Table 2: Parameters

\begin{tabular}{|c|c|}
\hline Parameter & Description \\
\hline$p_{c}$ & The positioning of each catenary node $[\mathrm{km}]$ \\
\hline$G_{n, n}$ & Real part of admittance matrix $[\mathrm{S}]$ \\
\hline$B_{n, n}$ & Imaginary part of admittance matrix $[j \cdot \mathrm{S}]$ \\
\hline$A, B, C$ & $\begin{array}{l}\text { Resistive force parameters } \text { Abrahamsson, 2008) for a } \\
\text { train }\left[15400 \mathrm{~N}, 279 \frac{\mathrm{N} \cdot \mathrm{s}}{\mathrm{m}}, 49.2 \frac{\mathrm{N} \cdot \mathrm{s}^{2}}{\mathrm{~m}^{2}}\right]\end{array}$ \\
\hline$m$ & Train mass $[1470000 \mathrm{~kg}] \quad \mathrm{m}^{2}$ \\
\hline$\theta^{50 ; g}$ & The no-load angle of the public grid [0 rad] \\
\hline$X_{q}^{50}$ & The short-circuit reactance of the public grid [0.15 p.u.] \\
\hline$U^{50 ; g}$ & The voltage at the public grid side $[1$ p.u.] of converter \\
\hline$Q_{50 ; g}$ & Reactive power consumption on the motoring side $[0$ p.u. \\
\hline$\#_{q}^{\text {conv }}$ & Number of converters per station $[6]$ \\
\hline$x_{q M}$ & $\begin{array}{l}\text { Motor-side inner reactance of rotary converter } \mathrm{Q} 48 / \mathrm{Q} 49 \\
{[0.22897 \text { p.u. }]}\end{array}$ \\
\hline$x_{q G}$ & $\begin{array}{l}\text { Generator-side inner reactance of rotary converter } \\
\text { Q48/Q49, including transformer [0.3036 p.u.] }\end{array}$ \\
\hline$F_{\max }$ & Maximal train tractive force $[275000 \mathrm{~N}]$ \\
\hline$a_{\max }$ & Maximal train acceleration $\left[0.85 \mathrm{~m} / \mathrm{s}^{2}\right]$ \\
\hline$r$ & Length of studied catenary section $[\mathrm{km}]$ \\
\hline$d^{\delta}$ & Length of each time step [minutes] \\
\hline$v_{\max }$ & Speed limit $[160 \mathrm{~km} / \mathrm{h}]$ \\
\hline$v_{\min }$ & The assumed slowest possible average speed $[80 \mathrm{~km} / \mathrm{h}]$ \\
\hline$U_{b}$ & Base voltage of power system $[16.5 \mathrm{kV}]^{\mathrm{x}}$ \\
\hline$U_{b 2}$ & Base voltage for tractive force curve $[15 \mathrm{kV}]$ \\
\hline$S_{b}$ & Base power of power system [5 MVA] \\
\hline$F_{\max }^{r}$ & Maximal possible running resistance of train $[\mathrm{N}]$ \\
\hline$F_{t, \delta}^{b, \max }$ & Maximal allowed braking force for train $t$ at time $\delta[\mathrm{N}]$ \\
\hline$v_{t, \delta}^{\max }$ & Maximal train speed for train $t$ at time $\delta[\mathrm{km} / \mathrm{h}]$ \\
\hline$p_{t, \delta}^{\iota, o}$ & Minimal train position for train $t$ at time $\delta[\mathrm{km}]$ \\
\hline$p_{t, \delta}^{\max }$ & Maximal train position for train $t$ at time $\delta[\mathrm{km}]$ \\
\hline
\end{tabular}


Table 3: Variables

\begin{tabular}{cl}
\hline Variable & Description \\
\hline$s_{t, \delta}$ & Is the train standing still? binary \\
$d_{t, \delta}$ & Is the train driving? binary \\
$b_{t, \delta}$ & Is the train braking? binary \\
$h_{t, \delta}$ & Has the train braked? binary \\
$\alpha_{t, \delta, c}$ & Share of train $t$ load assigned to catenary node $c$ SOS2 \\
$F_{t, \delta}^{r}$ & The resistive force of the train [N] \\
$v_{t, \delta}$ & The velocity of the train [km/h] \\
$p_{t, \delta}$ & The position of the train [km] \\
$U_{n, \delta}$ & The voltage level at each node [p.u.] \\
$U_{t, \delta}^{t}$ & The voltage level at each train [p.u.] \\
$\theta_{n, \delta}$ & The voltage angle at each node [rad] \\
$F_{t, \delta}^{u}$ & The used tractive force of train $t$ [N] \\
$F_{t, \delta}^{b}$ & The used braking force of train $t[\mathrm{~N}]$ \\
$P_{t, \delta}^{t}$ & The active power consumption of the train $[\mathrm{W}]$ \\
$Q_{t, \delta}^{t}$ & The reactive power consumption of the train [VAr] \\
$a_{t, \delta}$ & The acceleration of the train [m/s $\left.{ }^{2}\right]$ \\
$P_{n, \delta}^{n}$ & The net active power injection in node $n$ [p.u.] \\
$Q_{n, \delta}^{n}$ & The net reactive power injection in node $n$ [p.u.] \\
$P_{n, \delta}^{g}$ & The active power inflow to the RPSS at converter station $g$ [p.u.] \\
$Q_{n, \delta}^{g}$ & The reactive power inflow to the RPSS at converter station $g$ [p.u.] \\
$P_{n, \delta}^{d}$ & The active power consumption at node $n$ [p.u.] \\
$Q_{n, \delta}^{d}$ & The reactive power consumption at node $n$ [p.u.] \\
$\theta_{g, \delta}^{0}$ & The voltage angle at the public-grid side of converter node $g[\mathrm{rad}]$ \\
$\Psi_{g, \delta}$ & The phase-shift on the railway side of the converter node $g[\mathrm{rad}]$ \\
$z$ & The objective function \\
\hline &
\end{tabular}


assumed to be straight and flat. The maximal possible running resistance

$$
F_{\max }^{r}=A+B \frac{v_{\max }}{3.6}+C \frac{v_{\max }^{2}}{3.6^{2}}
$$

is defined by the running resistance at maximal allowed speed. The variables in Table 3 are bounded by

$$
\begin{aligned}
& 0 \leq \alpha_{t, \delta, c} \leq 1 \\
& 0 \leq F_{t, \delta}^{r} \quad \leq F_{\max }^{r} \\
& 0 \leq v_{t, \delta} \leq v_{t, \delta}^{\max } \\
& p_{t, \delta}^{\min } \leq p_{t, \delta} \quad \leq p_{t, \delta}^{\max } \\
& 0.5 \leq U_{n, \delta} \leq 1.1 \\
& 0.5 \leq U_{t, \delta}^{t} \quad \leq 1.1 \\
& \frac{-20}{180} \pi \leq \theta_{n, \delta} \quad \leq \frac{5}{180} \pi \\
& 0 \leq F_{t, \delta}^{u} \leq \begin{cases}F_{\max }, & \delta \leq \frac{r \cdot 60}{d^{6} v_{\min }} \\
0, & \delta>\frac{r \cdot 6}{d^{\delta} v_{\min }}\end{cases} \\
& 0 \leq F_{t, \delta}^{b} \quad \leq F_{t, \delta}^{b, \max } \\
& 0 \leq P_{t, \delta}^{t} \quad \leq\left\{\begin{array}{cl}
\frac{F_{\max } v_{\max }}{3.6}, & \delta \leq \frac{r \cdot 60}{d^{\delta} v_{\min }} \\
0, & \delta>\frac{r .60}{d^{\delta} v_{\min }}
\end{array}\right. \\
& 0 \leq Q_{t, \delta}^{t} \quad \leq 0 \\
& 0 \leq a_{t, \delta} \quad \leq 0, \delta>\frac{r \cdot 60}{v_{\min } d^{\delta}} \\
& -a_{\max } \leq a_{t, \delta} \leq \frac{F_{\max }}{m}, \delta \leq \frac{r \cdot 60}{v_{\min } d^{\delta}} \\
& -\frac{9 \#_{g}^{\text {conv }}}{4} \leq P_{g, \delta}^{g} \quad \leq \frac{9 \#_{g}^{\text {conv }}}{4} \\
& -\frac{9 \#_{g}^{\text {conv }}}{4} \leq Q_{g, \delta}^{g} \leq \frac{9 \#_{g}^{\text {conv }}}{4} \\
& 0 \leq P_{n_{n g}, \delta}^{g} \leq 0 \\
& 0 \leq Q_{n_{n g}, \delta}^{g} \leq 0 \\
& 0 \leq P_{c, \delta}^{d} \quad \leq 5 \\
& 0 \leq P_{n_{n c}, \delta}^{d} \leq 0 \\
& 0 \leq Q_{n, \delta}^{d} \quad \leq 0
\end{aligned}
$$




$$
\begin{array}{ll}
\frac{-20}{180} \pi \leq \theta_{g, \delta}^{0} & \leq \frac{5}{180} \pi \\
\frac{-20}{180} \pi \leq \Psi_{g, \delta} & \leq \frac{5}{180} \pi
\end{array}
$$

where

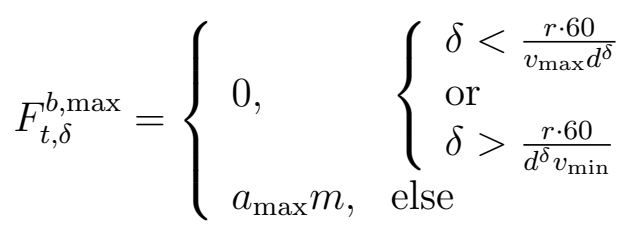

where

$$
v_{t, \delta}^{\max }= \begin{cases}v_{\max }, & \delta \leq \frac{r \cdot 60}{d^{\delta} v_{\min }}, \delta \neq 1 \\ 0, & \delta>\frac{r \cdot 60}{d^{\delta} v_{\min }}, \delta=1\end{cases}
$$

where

$$
\begin{aligned}
& p_{t, \delta}^{\min }= \begin{cases}0, & \delta \leq \frac{r \cdot 60}{d^{\delta} v_{\min }} \\
r, & \delta>\frac{r \cdot 60}{d^{\delta} v_{\min }}\end{cases} \\
& p_{t, \delta}^{\max }= \begin{cases}0, & \delta=1 \\
\frac{v_{\max }(\delta-1) d^{\delta}}{60}, & \left\{\begin{array}{l}
\delta \neq 1, \\
\delta<\frac{r \cdot 60}{v_{\max } d^{\delta}}
\end{array}\right. \\
r, & \delta \geq \frac{r \cdot 60}{v_{\max } d^{\delta}}\end{cases}
\end{aligned}
$$

and where the level values (values of the variables in the initial step of the iteration) of the variables are set to zero, except for the voltages which level values are set to 1 .

The term level value is another term for the variable values that the algorithm starts with. It is theoretically wiser from convergence and optimality points of view to chose a feasible start point for the solver, or a start point close to the expected optimal point. In this article however, the focus is not set on warm-starting the problems optimally.

The net injected powers $P^{n}$ and $Q^{n}$ are not bounded because by experience it is hard to find relevant bounds for them. The objective $z$ is not bounded either because a majority of the existing solvers for GAMS perform better with unbounded objectives (McCarl et al., 2012). 
The constraints of the optimization problem are

$$
\begin{aligned}
& a_{t, \delta}=\frac{F_{t, \delta}^{u}-F_{t, \delta}^{b}-F_{t, \delta}^{r}}{m} \\
& v_{t, \delta+1}=v_{t, \delta}+\frac{a_{t, \delta} d^{\delta} 3600^{2}}{1000 \cdot 60} \\
& p_{t, \delta+1}=p_{t, \delta}+\frac{v_{t, \delta} d^{\delta}}{60}+\frac{a_{t, \delta}\left(d^{\delta} 60\right)^{2}}{2000}, \delta \neq \Delta \\
& 0=\frac{v_{t, \delta} d^{\delta}}{60}+\frac{a_{t, \delta}\left(d^{\delta} 60\right)^{2}}{2000}, \delta=\Delta \\
& F_{t, \delta}^{r} \leq A+B \frac{v_{t, \delta}}{3.6}+C\left(\frac{v_{t, \delta}}{3.6}\right)^{2}+F_{\max }^{r} s_{t, \delta} \\
& F_{t, \delta}^{r} \geq A+B \frac{v_{t, \delta}}{3.6}+C\left(\frac{v_{t, \delta}}{3.6}\right)^{2}-F_{\max }^{r} s_{t, \delta} \\
& F_{t, \delta}^{r} \leq F_{\max }^{r}\left(1-s_{t, \delta}\right) \\
& b_{t, \delta-1} \leq b_{t, \delta}+s_{t, \delta} \\
& d_{t, \delta-1} \leq d_{t, \delta}+b_{t, \delta} \\
& F_{t, \delta}^{u} \leq d_{t, \delta} F_{\max } \\
& b_{t, \delta} \leq 1-10^{-3}+\frac{F_{t, \delta}^{b}}{a_{\max } m} \\
& b_{t, \delta} \geq-10^{-3}+\frac{F_{t, \delta}^{b}}{a_{\max } m} \\
& 1 \geq s_{t, \delta}+d_{t, \delta-1} \\
& 1 \geq s_{t, \delta}+d_{t, \delta} \\
& 1 \geq b_{t, \delta}+s_{t, \delta} \\
& 1 \geq b_{t, \delta}+d_{t, \delta} \\
& 1 \geq h_{t, \delta}+d_{t, \delta} \\
& h_{t, \delta} \geq \frac{b_{t, \delta}+h_{t, \delta-1}}{2} \\
& b_{t, \delta} \leq 1-\frac{h_{t, \delta-1}-b_{t, \delta-1}}{2} \\
& s_{t, \delta-1} \leq s_{t, \delta}+d_{t, \delta} \\
& s_{t, \delta-1} \leq 1-\left(\left(\frac{a_{t, \delta}}{a_{\max }}\right)^{2}+\frac{v_{t, \delta}}{v_{\max }}\right)
\end{aligned}
$$




$$
\begin{aligned}
& s_{t, \delta-1} \geq 10^{-3}-\left(\left(\frac{a_{t, \delta}}{a_{\max }}\right)^{2}+\frac{v_{t, \delta}}{v_{\max }}\right) \\
& F_{\max } \geq \frac{F_{t, \delta}^{u} v_{t, \delta}}{78} \\
& F_{\max } \frac{U_{t, \delta}^{t} U_{b}}{U_{b 2}} \geq \frac{F_{t, \delta}^{u} v_{t, \delta}}{78} \\
& 1=\sum_{c} \alpha_{t, \delta, c} \\
& p_{t, \delta}=\sum_{c} \alpha_{t, \delta, c} p_{c} \\
& P_{t, \delta}^{t}=F_{t, \delta}^{u} \frac{v_{t, \delta}}{3.6} \\
& P_{n, \delta}^{d}=\sum_{t} \alpha_{t, \delta, c} P_{t, \delta}^{t} \frac{10^{6}}{S_{b}} \\
& U_{t, \delta}^{t}=\sum_{c} \alpha_{t, \delta, c} U_{c, \delta} \\
& P_{n, \delta}^{n}=U_{n, \delta} \sum_{n_{2}} U_{n_{2}, \delta}\left(G_{n, n_{2}} \cos \left(\theta_{n, \delta}-\theta_{n_{2}, \delta}\right)+\right. \\
& \left.+B_{n, n_{2}} \sin \left(\theta_{n, \delta}-\theta_{n_{2}, \delta}\right)\right) \\
& Q_{n, \delta}^{n}=U_{n, \delta} \sum_{n_{2}} U_{n_{2}, \delta}\left(G_{n, n_{2}} \sin \left(\theta_{n, \delta}-\theta_{n_{2}, \delta}\right)-\right. \\
& \left.-B_{n, n_{2}} \cos \left(\theta_{n, \delta}-\theta_{n_{2}, \delta}\right)\right) \\
& 0=P_{n, \delta}^{g}-P_{n, \delta}^{d}-P_{n, \delta}^{n} \\
& 0=Q_{n, \delta}^{g}-Q_{n, \delta}^{d}-Q_{n, \delta}^{n} \\
& \theta_{g, \delta}^{0}=\theta^{50 ; g}-\frac{1}{3} \arctan \left(\frac{X_{g}^{50} \cdot P_{g, \delta}^{g}}{\left(U^{50 ; g}\right)^{2}+X_{g}^{50} \cdot Q_{50 ; g}}\right) \\
& \Psi_{g, \delta}=-\frac{1}{3} \arctan \left(\frac{x_{q M} P_{g, \delta}^{g}}{\#_{g}^{\operatorname{conv}}\left(U^{50 ; g}\right)^{2}+x_{q M} Q_{50 ; g}}\right)- \\
& -\arctan \left(\frac{x_{q G} P_{g, \delta}^{g}}{\#^{\text {conv }}\left(U_{g, \delta}\right)^{2}+x_{q G} Q_{g, \delta}^{g}}\right) \\
& \theta_{g, \delta}=\theta_{g, \delta}^{0}+\Psi_{g, \delta}
\end{aligned}
$$




$$
\begin{aligned}
U_{g, \delta} & =1-\frac{Q_{g, \delta}^{g} S_{b}}{\#^{\operatorname{conv}} \cdot 20 \cdot U_{b}} \\
z & =\sum_{t, \delta}\left(p_{t, \delta}^{\max }-p_{t, \delta}\right) d^{\delta} .
\end{aligned}
$$

\subsection{Modeling Description}

Given the catenary type, catenary lengths, system topography, the number and placement of desired electrical nodes (i.e. a discrete sampling of the catenary), the admittance matrix is created. It has been assumed that the trains consume no reactive power, that the constant power part of the tractive force curve starts at $78 \mathrm{~km} / \mathrm{h}$ and that the constant power part is linearly reduced as a function of pantograph voltage level, for voltage levels below $15 \mathrm{kV}$. The tractive force curve used in the paper is illustrated in Figure 1. Moreover, auxiliary power load and internal train losses are neglected.

The electrical, physical, and computational explanations of the constraints are as follows:

The train acceleration is defined in (37), the train velocity in (38), and the train position in (39)- 40 , where 40 is a boundary condition for the last time step in the model.

The running resistance of the trains is defined by equations 41)-43. These three make sure that the running resistance is 0 for a train that is standing still, i.e. when $s_{t, \delta}$ is valued 1 , and equals the running resistance polynomial (Lukaszewicz, 2001) otherwise.

Still standing trains are defined by equations (57)-(58). In other words, trains are defined to be standing still if and only if they have no acceleration and no speed.

A train can only brake at one time step if it either brakes or stands still in the next consecutive time step, c.f. equation (44). Note that this is a, for practical purposes, undesired constraint since trains in real traffic on main lines often encounter various speed limits and may then consequently have to brake and speed-up alternately. The constraint (44) will have to be modified or removed in future, more realistic models, c.f. the discussion about this in Section 6.3.

Moreover, a train can only drive if it drives or brakes in the next consecutive time step, as stated in (45). This precludes the possibilities of trains slowly stopping just due to running resistance. It implicitly says that the only allowed way to stop the train is by braking, since it is not allowed to stand still right after driving. 


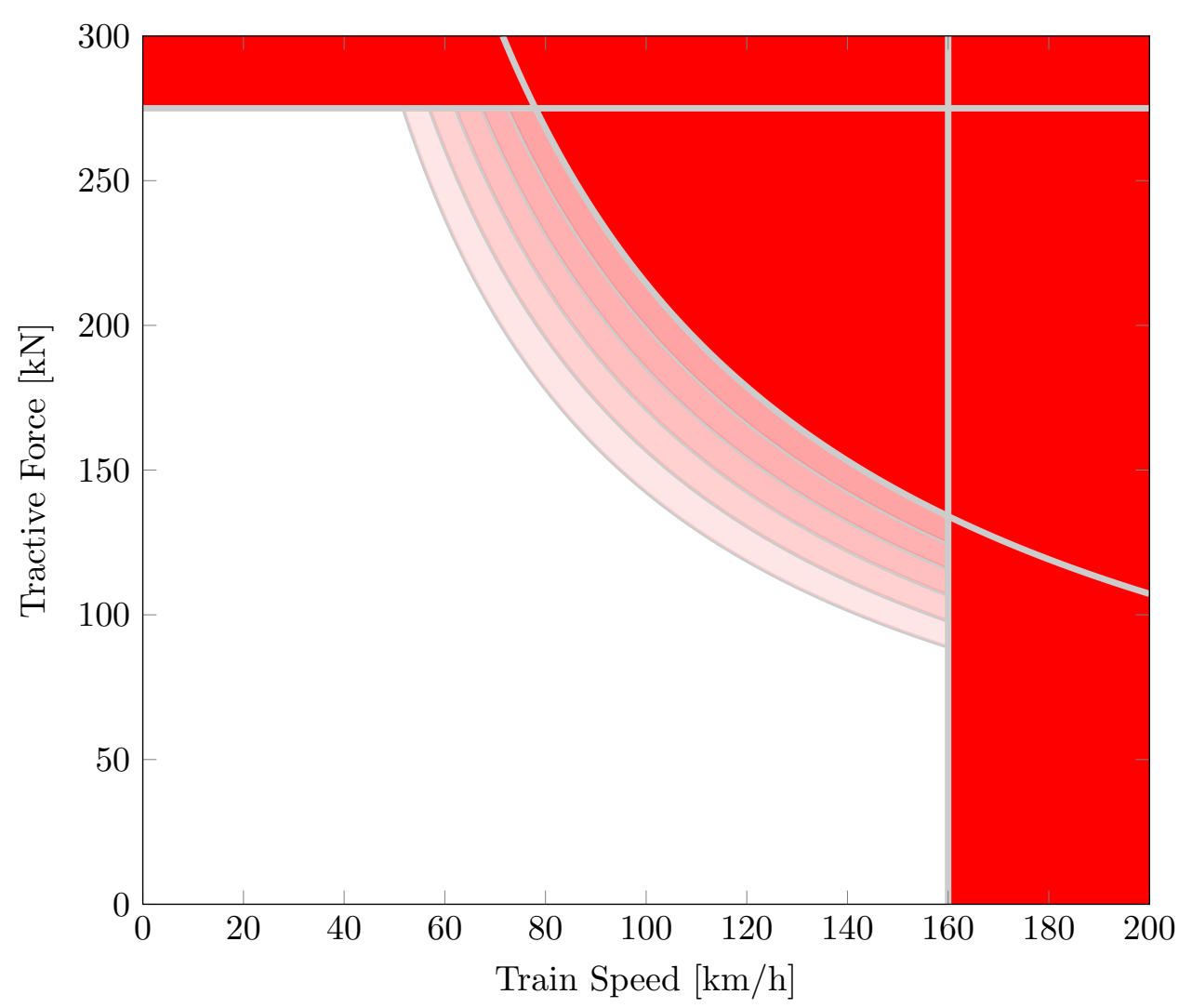

Figure 1: Tractive force curve description. Red areas are always forbidden. Horizontal line represents the constant force part of the curve, c.f. equation (18). Vertical line represents the speed limit part of the curve, c.f. equation (13). The curves inversely proportional to train speed represents the constant power region of the curve. The first of the constant power curves, the one right below the purely red area represents catenary voltage levels on and above $15 \mathrm{kV}$. The following curves, represents maximal tractive forces for $14 \mathrm{kV}, 13 \mathrm{kV}, 12 \mathrm{kV}, 11 \mathrm{kV}$, and $10 \mathrm{kV}$, respectively. The forbidden tractive force curve area increases with decreasing voltage levels. The voltage-dependency is a continuous and piecewise smooth function modeled by equations (59)- 600 .

Equation (46) makes sure that the train tractive force is nonzero only when the train is driving. By similar reasons, equations (47)-(48) make sure that the braking force is nonzero if and only if the train is braking. 
A train cannot stand still in one time step right after a time step of driving, expressed in (49). Trains can never stand still and drive at the same time, c.f. (50); brake and stand still simultaneously, c.f. (51); brake and drive, c.f. (52); or drive and have been braking at the same time, c.f. (53).

Note that in Table 3 there is one binary variable indicating that the train is braking at the moment, and there is another variable indicating that the trains has been braking either at the time studied, or sometime before. In (54) it is declared that if a train has been braking at a certain time step $\delta-1$ or if it braked in time step $\delta$, it has been braking in the $\delta$ time step. Additionally, according to (55) a train cannot brake at a time step if it has been braking and stopped braking, to avoid pumping brakes and probably undesired local optima. A train can only stand still at a time step if it stands still or drives in the following time step, c.f. (56).

Equation (59) illustrates the constant power part of the tractive force curve, whereas (60) illustrates the constant power part affected by further reductions due to catenary voltage drops below nominal level at the pantograph. The tractive force curve is illustrated graphically in Figure 1 .

Equation (61) defines the SOS2 variables $\alpha_{t, \delta, c}$. Equation 62 projects the actual train position on the closest pair of catenary nodes, or in other words, defines the nonzero elements of the SOS2 variables. Tractive power is calculated by equation (63) whereas the tractive power is projected on the catenary nodes into electrical power by (64). Train (pantograph) voltages are in an analogous but reverse fashion computed as a weighted average of the voltages in the pair of neighboring catenary nodes by (65).

Active power flow is computed in (66), and reactive power flow in (67), whereas active power mismatch equations are stated in (68), and the reactive power mismatch in 69 ).

The converter stations are modeled in equations (70)- (73). Equation (70) models the public-grid-side phase shift due to converter loading, 71 represents the corresponding phase shift on the railway-grid-side of the converter, whereas (72) merely summarizes the phase shifts in order to determine the actual voltage angle on the railway side, and (73) models the voltage regulation (sometimes denoted voltage compounding) on the railway side of the converter station.

Last, but not least, the objective function (74), to be minimized, is determined by the difference between the trains destination position and its actual positions. That kind of objective forces the train to arrive, not only as fast as possible at its final destination, but actually as far as possible at 
every time step.

\section{Numerical Examples and Results}

In all the studies made, the catenary nodes were located equidistantly. The model as such does however allow non-uniform spatial sampling of the catenary.

\subsection{General Results}

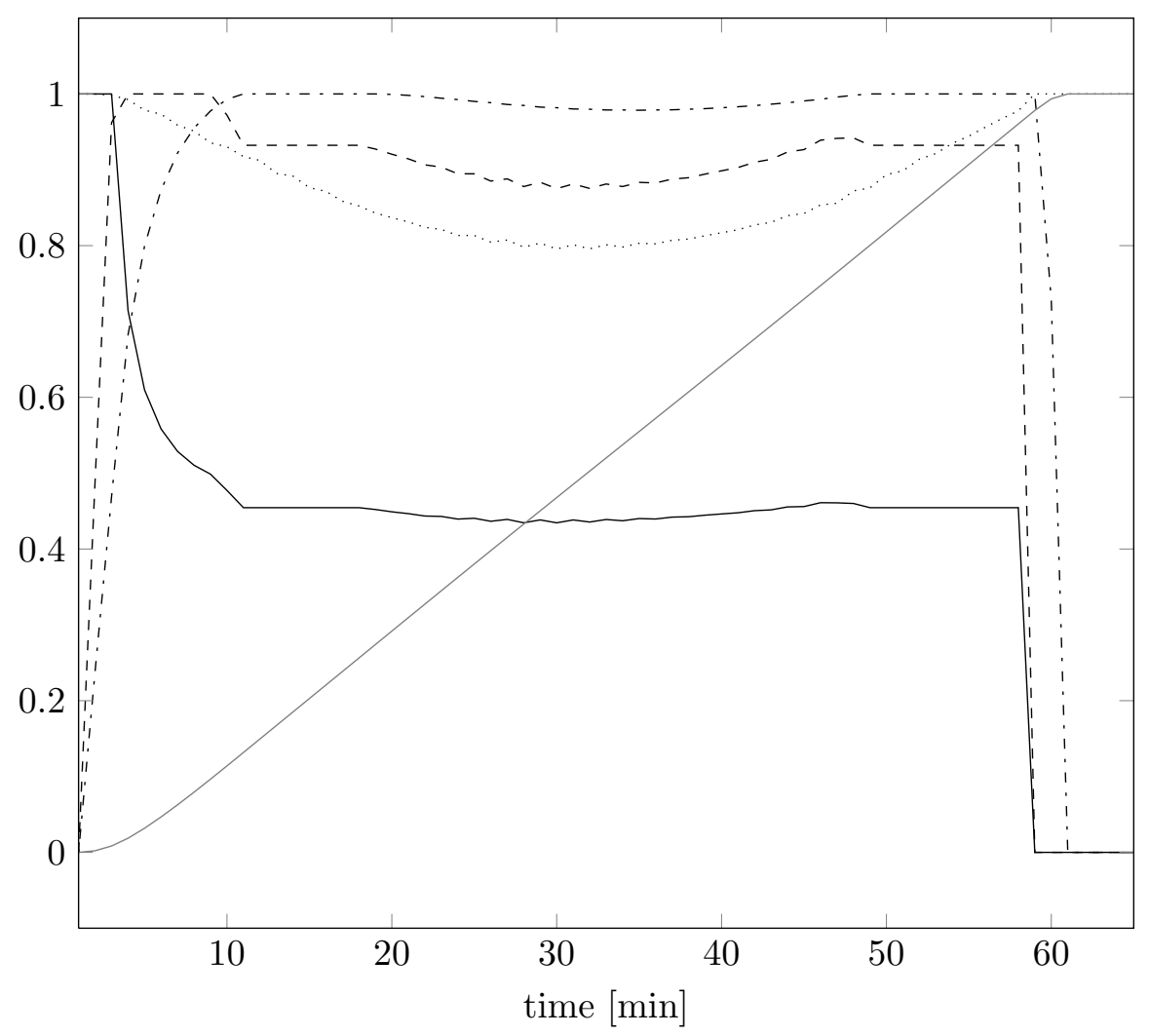

Figure 2: Electrical and mechanical properties of the train plotted against time. All the curves are normalized. Their descriptions and their normalization factors are stated in Table 4 .

This section is mainly for showing the functionality of the model presented. In this section, the same system is being studied, and the figures 2 , 


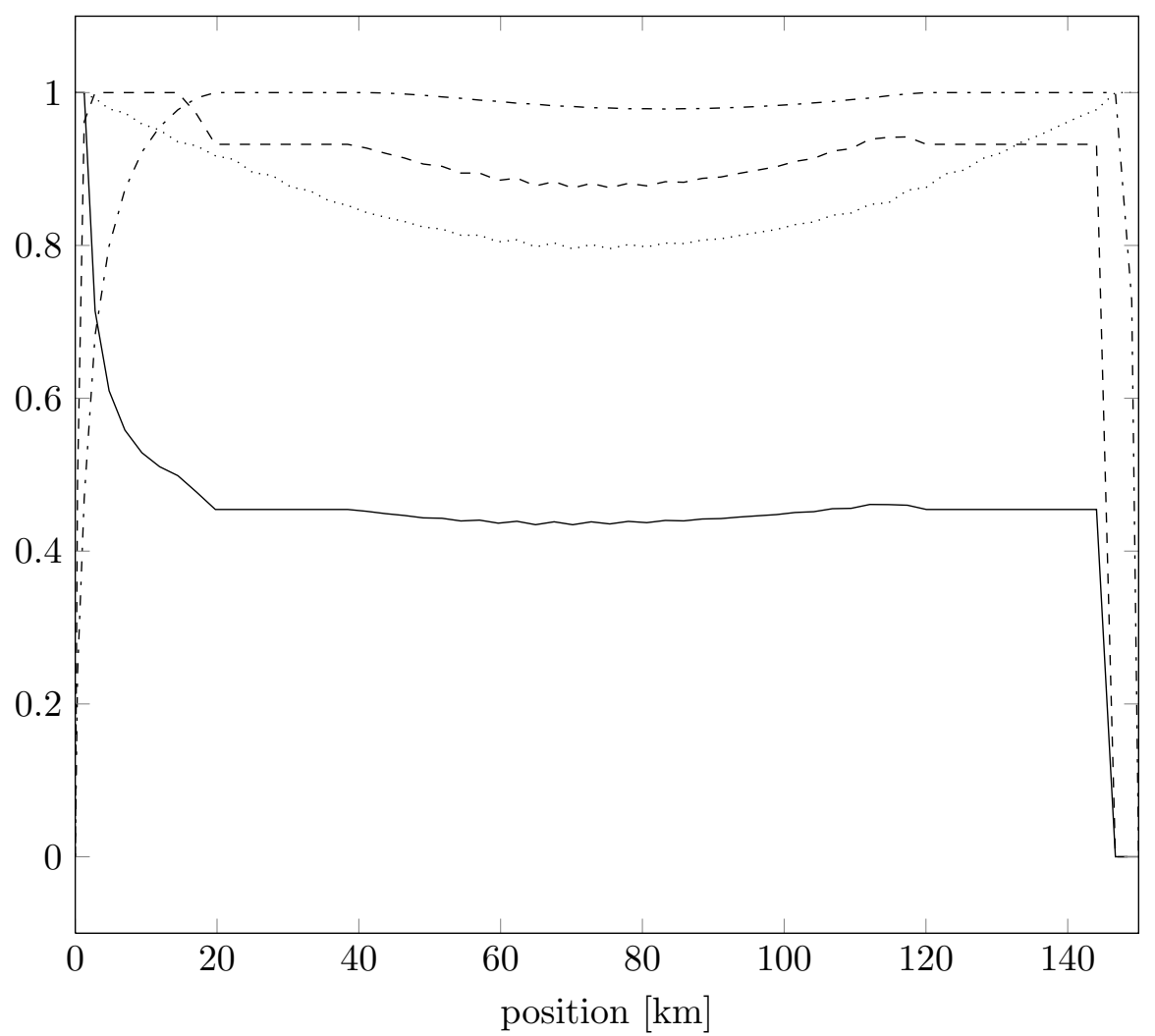

Figure 3: Electrical and mechanical properties of the train plotted against position. All the curves are normalized. Their descriptions and their normalization factors are stated in Table 4.

3, and 14 all contain curves normalized in the same fashion. The normalization is presented in Table 4. The examples are all taken from studies with a $150 \mathrm{~km}$ BT catenary section, 1 train, 1 minute time step length, and 31 catenary nodes.

In Figure 2 the resulting curves of tractive force, pantograph voltage, velocity, position, and power consumption as functions of time are displayed, whereas tractive force, pantograph voltage, velocity, and power consumption are presented as functions of train position in Figure 3.

Studying figures 2 and 3 , one can see that there are two flat levels of the traction power curve, the higher one represents the maximal power limitation of the train, $5.958 \mathrm{MW}$, computed by the upper limit of the constant power 
Table 4: Description of the normalizations being done in figures 2, 3, and 14 . These figures present case studies made in a $150 \mathrm{~km}$ BT catenary section, with 1 train, using 1 minute time step lengths, and 31 catenary nodes.

\begin{tabular}{llll}
\hline Technical entity & Label & Graphically represented by & Normalized by \\
\hline Train tractive force & $F^{u}$ & A solid black line & $275 \mathrm{kN}$ \\
Train voltage & $U^{t}$ & A dotted black line & $16.5 \mathrm{kV}$ \\
Train velocity & $v$ & A dash-dotted black line & $160 \mathrm{~km} / \mathrm{h}$ \\
Train active power & $P^{t}$ & A dashed black line & $5.958 \mathrm{MW}$ \\
consumption & & A solid dark grey line & $150 \mathrm{~km}$ \\
Train position & $p$ & & \\
\hline
\end{tabular}

constraint

$$
\frac{F_{\max } \cdot 78}{3.6}=5.598 \mathrm{MW}
$$

whereas the lower one, at $5.555 \mathrm{MW}$ originates from the running resistance at the speed limit

$$
\frac{v_{\max }}{3.6}\left(15400+279 \frac{v_{\max }}{3.6}+49.2\left(\frac{v_{\max }}{3.6}\right)^{2}\right)=5.555 \mathrm{MW},
$$

which is the special case of the maximal tractive force considering the speed limit, when already traveling at the maximal speed. The general function describing the speed-limit-imposed maximal tractive force is

$$
F_{v_{\max }}=\frac{m \cdot\left(v_{\max }-v\right) \cdot 1000 \cdot 60}{d^{\delta} \cdot 3600^{2}}+F^{r}
$$

and is illustrated graphically in figures 4 and 5 .

The lowest value of the tractive power, due to catenary voltage drops and reduced tractive performance, is $5.214 \mathrm{MW}$. That occurs in Figure 2 right after 30 minutes of traveling, and in Figure 3 after $75 \mathrm{~km}$ of traveling, i.e. in the middle of the catenary section.

In Figure 4 the bounding limiting tractive force functions are displayed zoomed up for the first traveled $22 \mathrm{~km}$ of the train. For the first $1 \mathrm{~km}$ the constant force part of the tractive force curve, c.f. (18), is actively bounding the tractive force. Thereafter, between $2 \mathrm{~km}$ and $16 \mathrm{~km}$, the constant power region, c.f. (59), of the tractive force curve is active. The constant speed part 


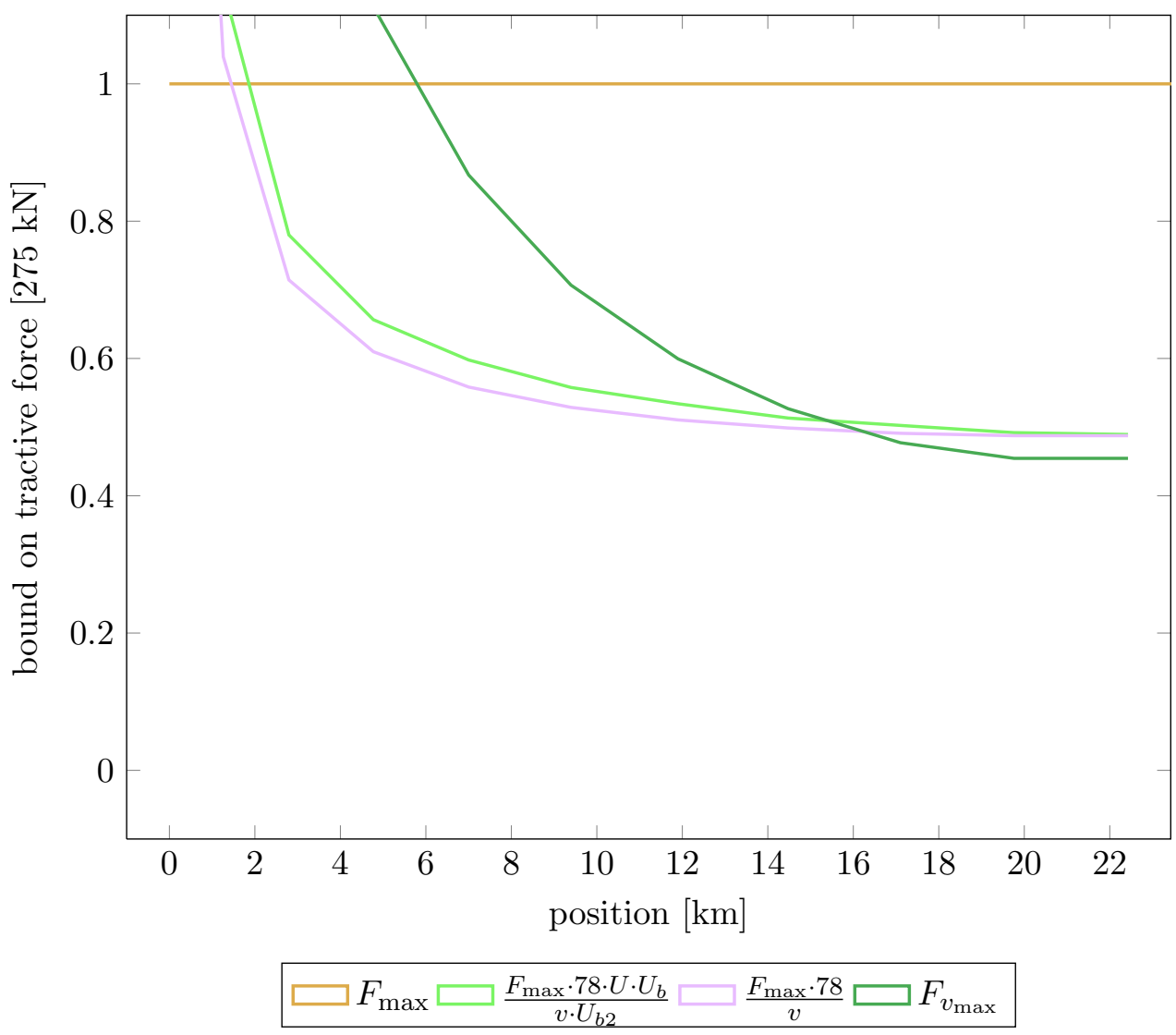

Figure 4: Bounding limiting train tractive forces. Zoomed at the first $22 \mathrm{~km}$ of the trip, in a 31 nodes, $150 \mathrm{~km}$ BT system.

of the bounds on the tractive force becomes active from $16 \mathrm{~km}$ on and into the next, un-zoomed figure, Figure 5, where the bounding force functions are illustrated for the entire trip. The speed-limit part, $F_{v_{\max }}$ is neither explicitly a variable bound, nor a functional constraint in the model, just an implicit bound originating from the speed limit, c.f. (77). The speed-limit is the active bound on used tractive force up to the train has travelled $40 \mathrm{~km}$, c.f. Figure 5. Thereafter, the catenary voltage actively limits, c.f. (60), the tractive force up to $120 \mathrm{~km}$ of the section has been passed by the train, which is illustrated in Figure 5. For the rest of the trip, from the $120 \mathrm{~km}$ position until braking has started, the speed limit is once again the actively bounding limit on the train tractive force.

In Figure 6, the voltage levels of all nodes for all time steps in this case 


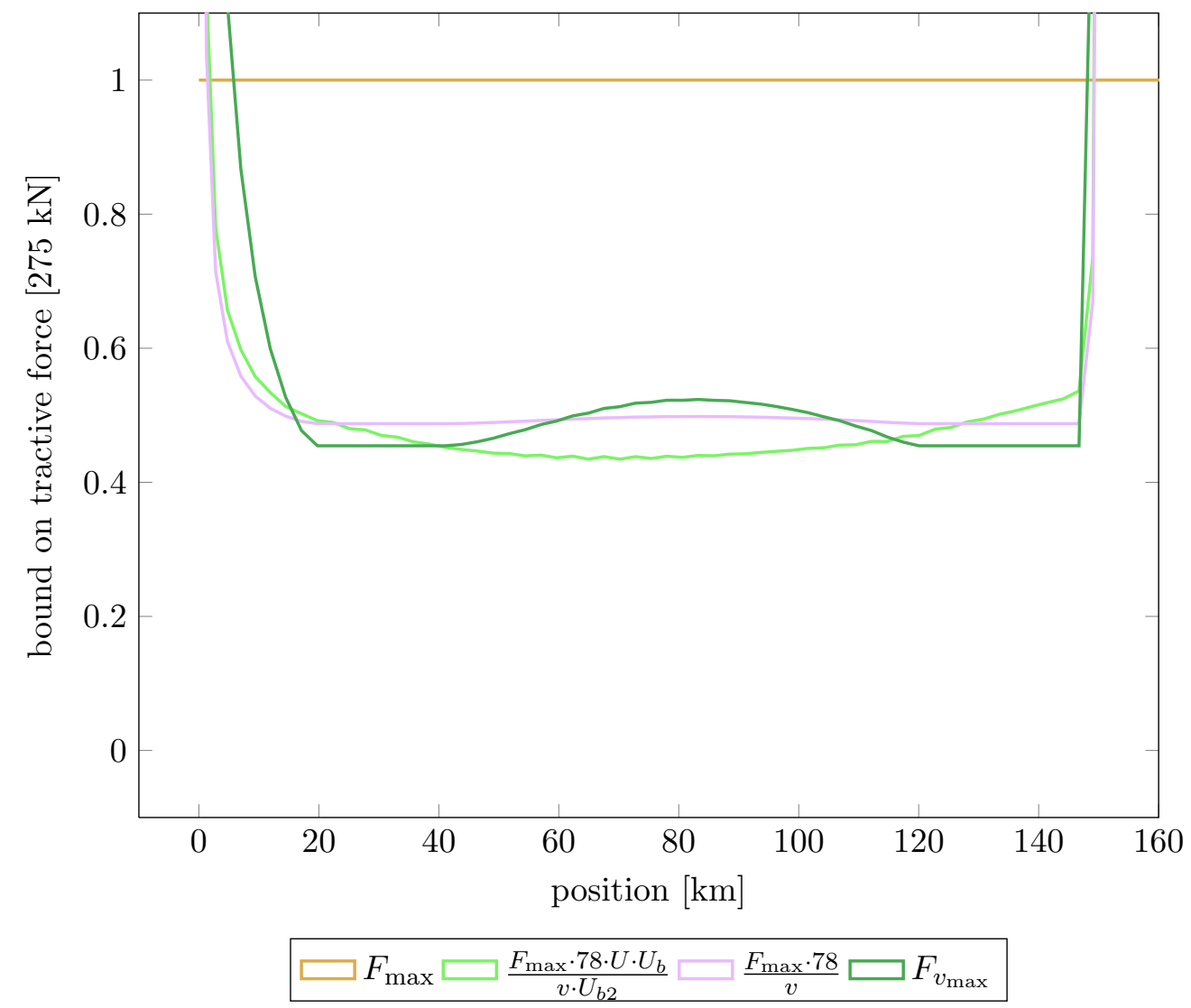

Figure 5: Bounding limiting train tractive forces. The entire trip, in a 31 nodes, $150 \mathrm{~km}$ BT system.

study are illustrated in a color matrix.

In order to further illustrate the functioning of railway electric traction system operation, and particularly the functioning of this presented model, the tractive force, $F^{u}$, and the resistive force, $F^{r}$, are plotted together in Figure 7. $F^{u}$ and $F^{r}$ are equal when the speed limit is actively bounding $F^{u}$. $F^{u}$ is larger than $F^{r}$ before reaching the speed limit. When entering the area where the catenary voltage limits the tractive force of the train, the resistive force surpasses the tractive force, wherefore the train slows down. When passing the $75 \mathrm{~km}$ location, i.e. the power section midpoint, the tractive force starts to exceed the resistive force once again and the train speeds gradually up to the speed limit. When the speed limit is reached, the resistive force and the tractive force equals each other once again. In the short final braking 


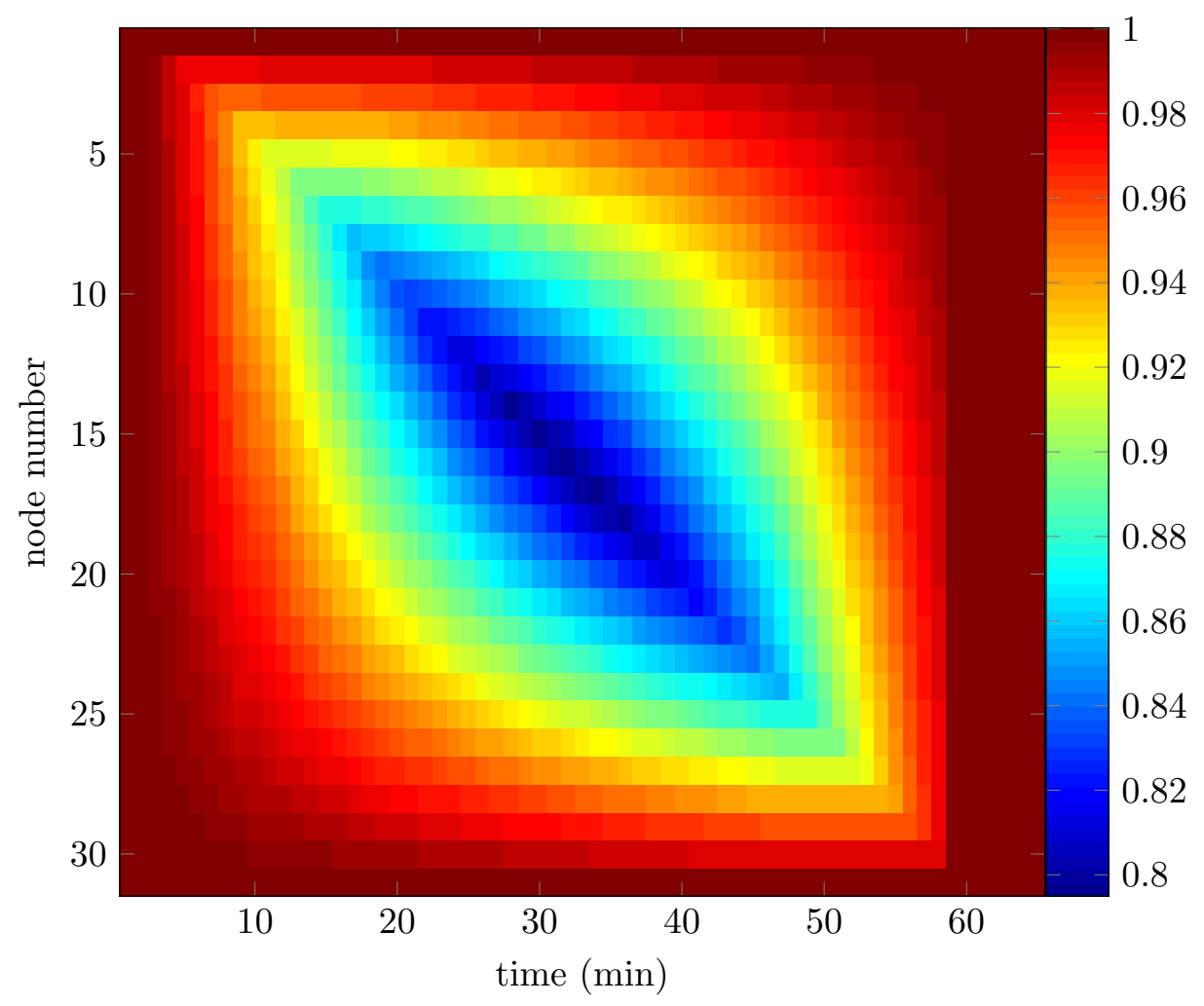

Figure 6: Voltages at the catenary nodes. All 31 nodes, plotted over 65 minutes, whereas the train trip lasts for 60 minutes. Voltage levels in p.u. with base voltage $16.5 \mathrm{kV}$. The catenary node voltages are illustrated over time in a color matrix, where the top values in dark red represents 1.000 p.u. and where the lowest value is $0.794 \mathrm{p} . \mathrm{u}$. and represented by dark blue. The lowest voltage is when the train is in the middle of the catenary section. For each train and time step, the lowest voltage is where the train is located.

mode, resistive force exceeds the tractive force until the train has stopped and they once again equal each other.

The edginess of the $F^{u}$ curve in Figure 7 is a result of time discretization steps of $1 \mathrm{~min}$, and of the fact that acceleration in time step $\delta$ affects the speed in time step $\delta+1$, c.f. (38), which in turn in time step $\delta+1$ affects the maximal possible tractive force function, c.f. (60). Since the speed is about $160 \mathrm{~km} / \mathrm{h}$, and the time step is $1 \mathrm{~min}$, the spatial discretization imposed by time discretization will be about $2 \frac{2}{3} \mathrm{~km}$. In Figure 7 it takes about 8 


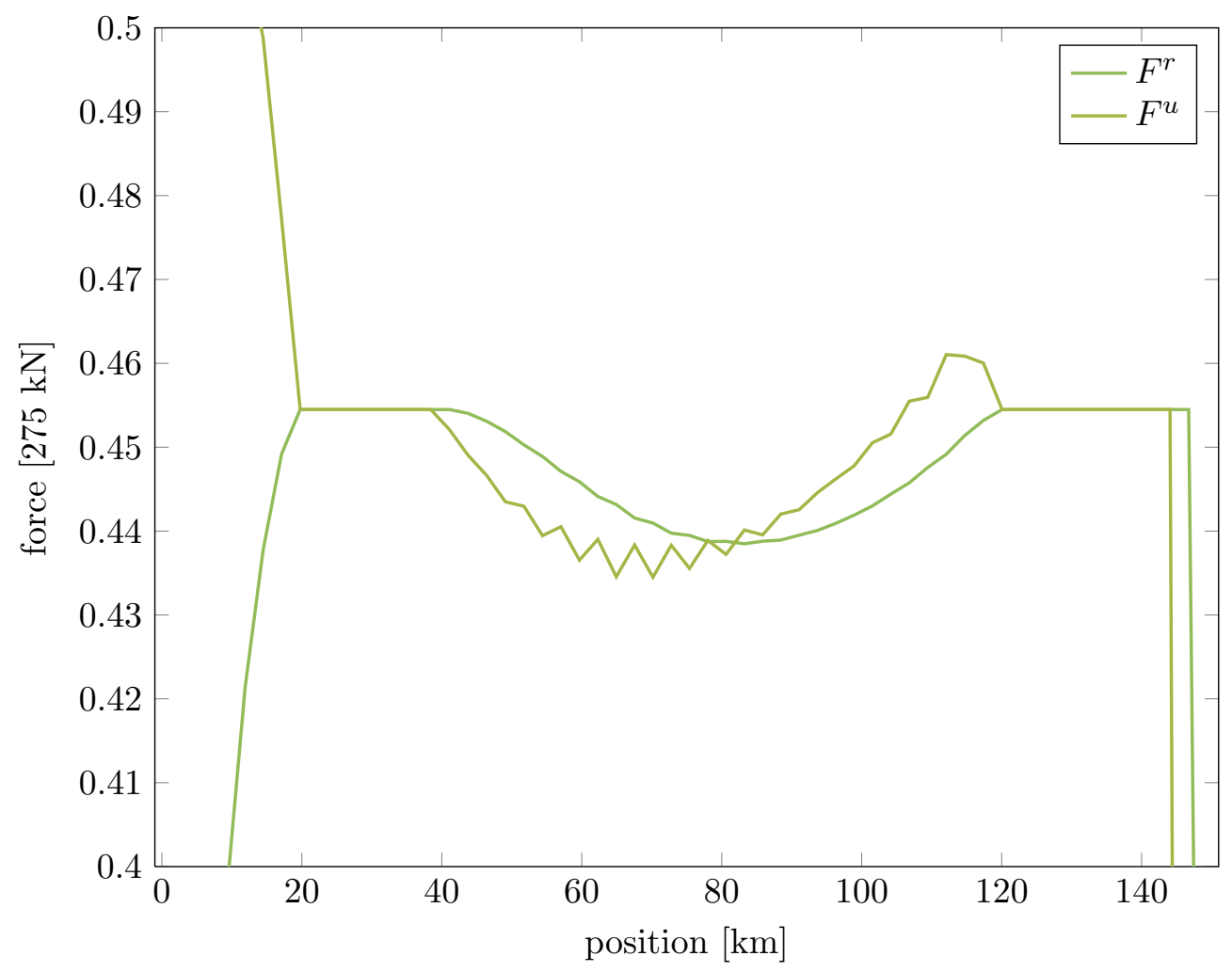

Figure 7: The used tractive force, $F^{u}$, and the resistive force, $F^{r}$, plotted for comparison. When speed is constant, the forces equal each other. When the voltage drops slow down the train, the resistive force exceeds the tractive force. After passing the power supply system section midpoint, the train is slowly able to regain its former speed. A 31 nodes, $150 \mathrm{~km}$ BT system section, with 1 train, and 1 minute time step case was studied.

crenatures to reach $20 \mathrm{~km}$, which justifies the conjecture of time discretization causing the edges.

\subsection{Illustrations of the application of the SOS2 variable}

In this section, the purpose is to graphically clarify how the optimal solutions regarding the SOS2 variable might look. The case studied in this section is a $60 \mathrm{~km}$ section, modeled with 40 electrical nodes, 1 train, and using 0.5 minutes time steps.

In Figure 8, the sub-matrix of $\alpha$ representing the one and only train 


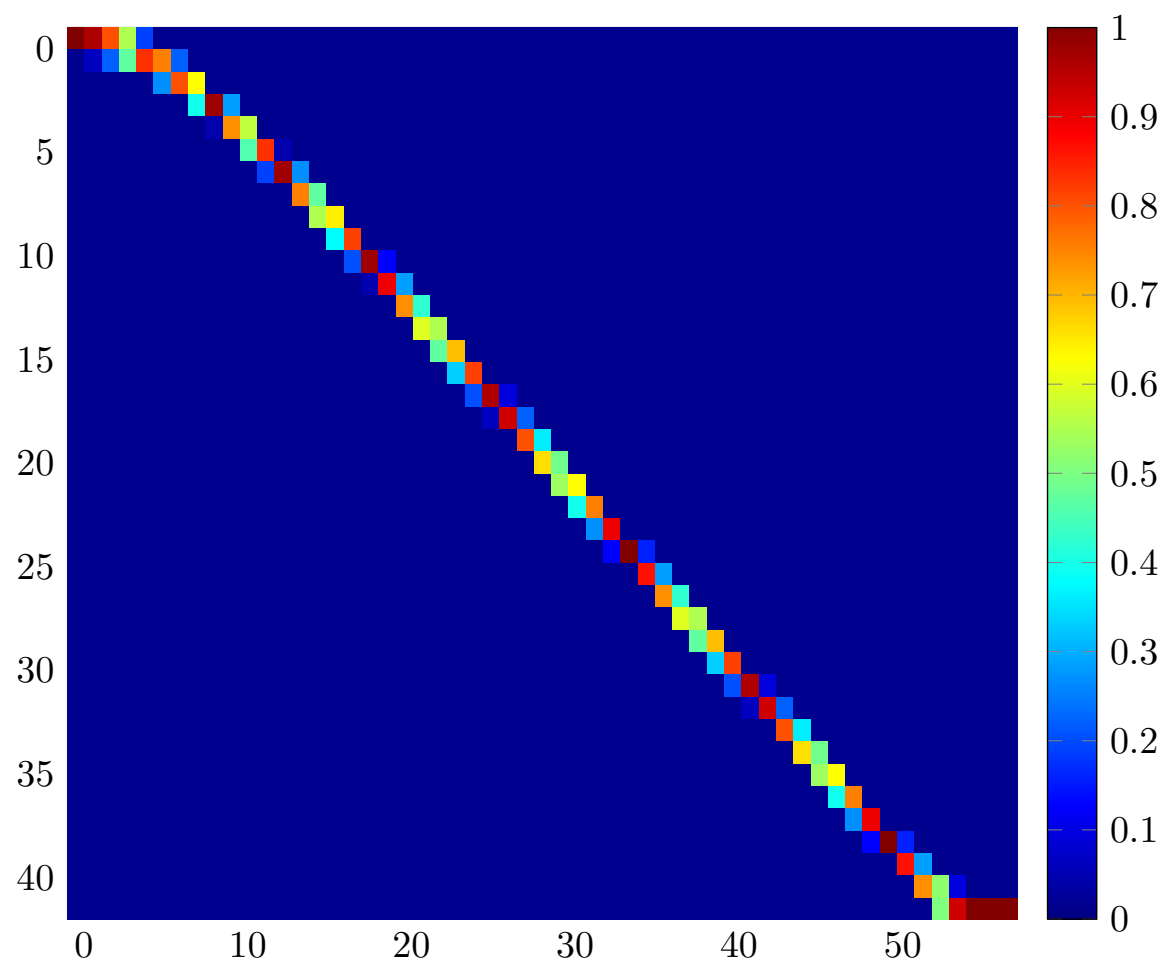

Figure 8: The numerical values of the weighting variable $\alpha$. Horizontal axis represents time steps, whereas vertical axis represents node numbers. Note that along the vertical axis, along the catenary for a given time step, the train is never represented by more than two nodes - the two most adjacent ones. Along the horizontal axis however, the train can very well be represented by the same nodes for many time steps. This is completely realistic if the train drives slowly.

studied is plotted. Maximally two elements are nonzero for each time step, the sums of the paired elements for each time step are always 1 .

Figure 9 illustrates how the train power consumption, $P_{t ; \delta}^{t}$, is distributed and shared between the two most adjacent electrical nodes.

In Figure 11 it is illustrated how the voltage levels of the two nodes most adjacent to the train are weighted together using $\alpha$ to approximate the pantograph voltage level of the train. 


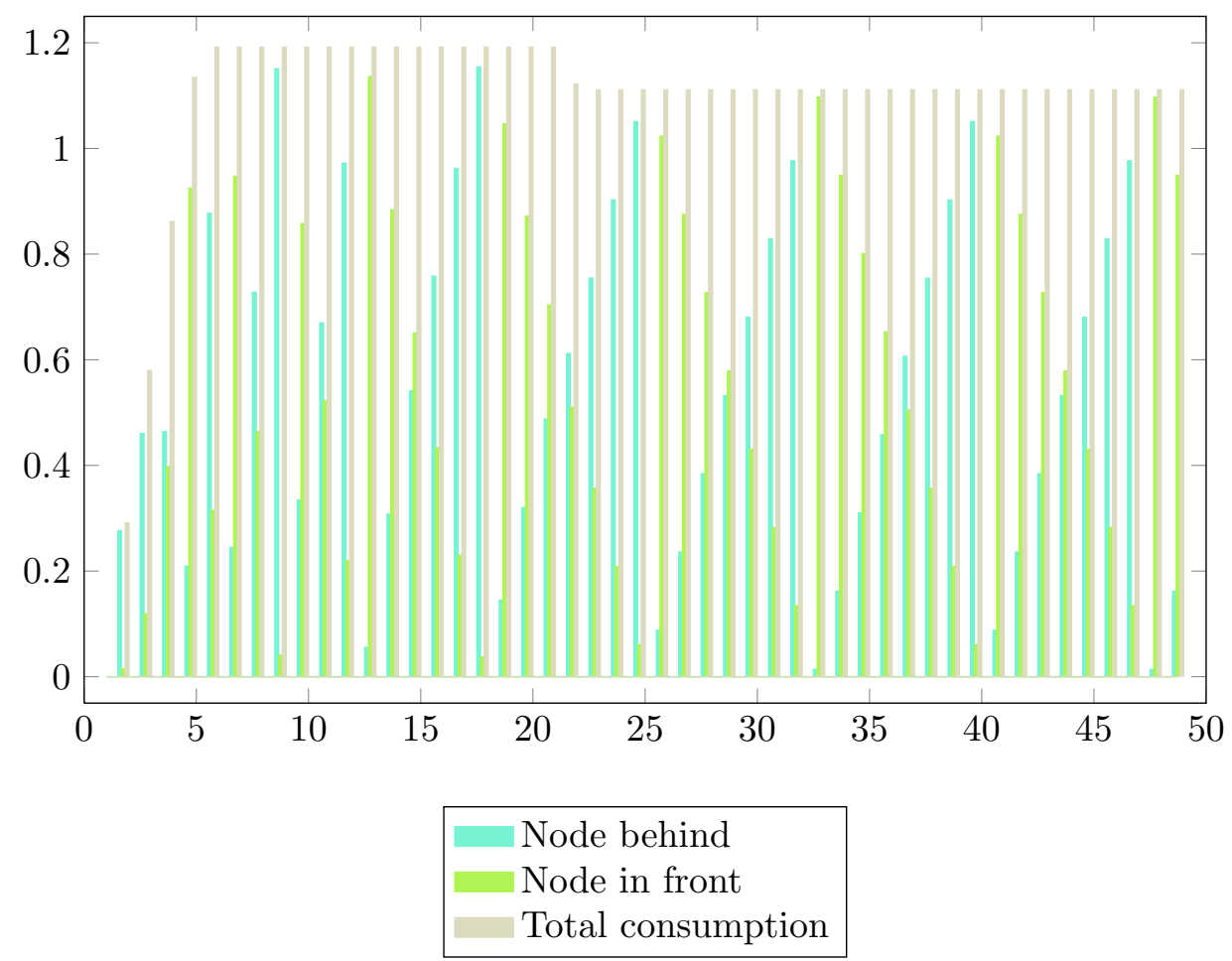

Figure 9: Illustration by bar diagram, how the actual train power is subdivided as nodal loads to the two nodes most adjacent to the train. Horizontal axis represents time steps, whereas vertical axis represents nodal active power consumption $P_{n, \delta}^{d}$, and train active power consumption $P_{t ; \delta}^{t}$, respectively. All powers are expressed in p.u., with 5 MVA base power. A zoom on the first 11 time steps is available in Figure 10 to further clarify.

\subsection{The impact of time step size variations}

Since the problem is cumbersome to solve on personal computers when using small time step sizes and catenaries longer than $100 \mathrm{~km}$, a $50 \mathrm{~km}$ section is compared for time step sizes of $0.1,0.2,0.5,1$, and 2 minutes respectively and 10 equidistant catenary nodes, c.f. Table 6 .

\subsection{The impact of catenary node density}

For the 1 minute time steps, 1 train, and $50 \mathrm{~km}$ catenary case, the train traveling time results are exactly equal regardless of the number of catenary nodes. This is because there is no need for detailed power system models if the grid is strong in comparison to the load, and energy or power consumption 


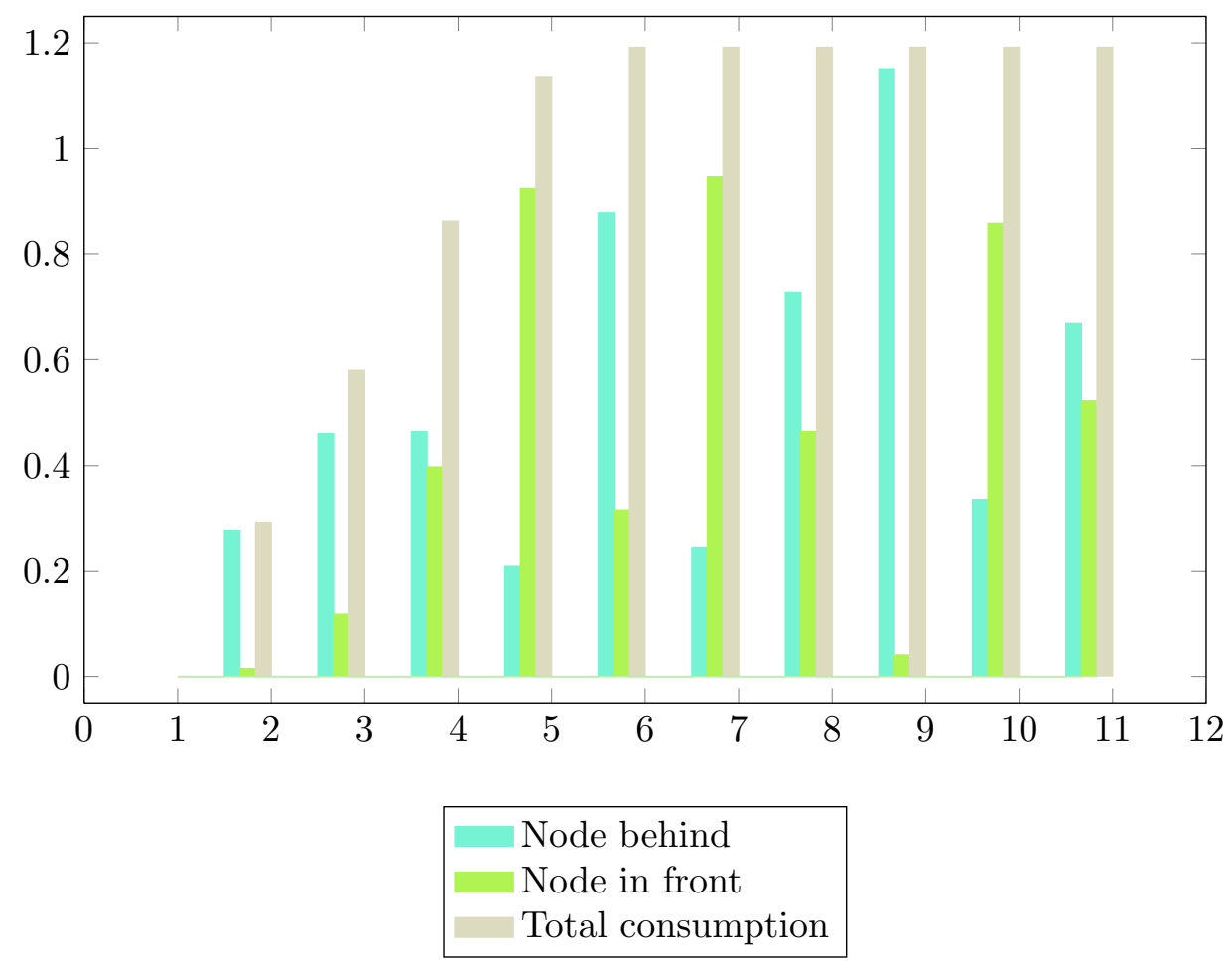

Figure 10: Illustration by bar diagram, how the actual train power is subdivided as nodal loads to the two nodes most adjacent to the train. Horizontal axis represents time steps, whereas vertical axis represents nodal active power consumption $P_{n, \delta}^{d}$, and train active power consumption $P_{t ; \delta}^{t}$, respectively. All powers are expressed in p.u., with 5 MVA base power. A zoom on the first 11 time steps. The corresponding $\alpha$ values are listed in Table 5 .

is not studied. In Figure 12a, the number of catenary nodes is varied from 3 to 53 and there is a clear linear trend in the computation times for increased number of nodes.

For the 1 minute time steps, one single train, and $150 \mathrm{~km}$ catenary case, the train traveling time results slowly converge to an objective function value slightly greater than 410 when the number of catenary nodes exceed 35 , c.f. Figure 13. The trend is that for increased number of nodes, the traveling times increase. This can be explained with that the voltage drops are with higher nodal resolution of the power system not evened out as much by the linear bi-nodal interpolation used. The computational times increase dramatically and then settle for around $10^{5}$ seconds illustrated in Figure $12 \mathrm{~b}$. 
Table 5: Numerical values of the SOS2 variable $\alpha$ for the 11 first time steps in the study presented in Section 4.2 .

\begin{tabular}{lllllll}
\hline & $\delta=1$ & $\delta=2$ & $\delta=3$ & $\delta=4$ & $\delta=5$ & $\delta=6$ \\
\hline Node behind & 1 & 0.94834 & 0.79395 & 0.53848 & 0.18465 & 0.73609 \\
Node in front & 0 & 0.051655 & 0.20605 & 0.46152 & 0.81535 & 0.26391 \\
\hline & $\delta=7$ & $\delta=8$ & $\delta=9$ & $\delta=10$ & $\delta=11$ & \\
\hline Node behind & 0.20525 & 0.61063 & 0.96584 & 0.28049 & 0.56186 & \\
Node in front & 0.79475 & 0.38937 & 0.034156 & 0.71951 & 0.43814 & \\
\hline
\end{tabular}

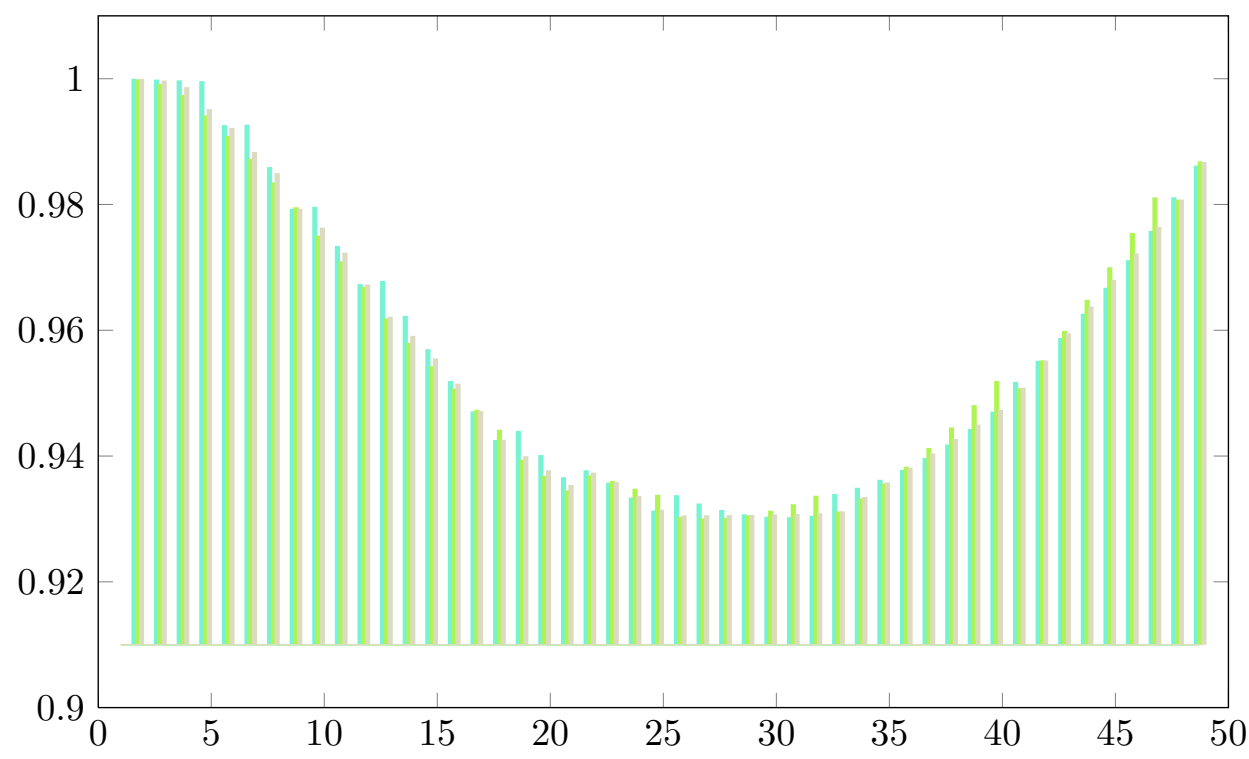

Node behind

Node in front

Average Voltage

Figure 11: Illustration by bar diagram, how the catenary voltages of the two most adjacent nodes to the train are weighted together, using $\alpha$, in order to determine the voltage level at the pantograph. Horizontal axis represents time steps, whereas vertical axis represents nodal voltage levels and weighted train voltage levels in p.u., with $16.5 \mathrm{kV}$ base voltage. 
Table 6: The impact of discretization time step on computation times and objective values. The results in the table are from a $50 \mathrm{~km}$ section, with 10 equidistant catenary nodes, and one single train trafficking. The time step lengths varies from 2 minutes down to 0.2 minutes (which is $12 \mathrm{~s}$ ).

\begin{tabular}{llc}
\hline Time Step length (min) & Objective values & Computation times (s) \\
\hline 2 & 114.33 & 11.043 \\
1 & 126.08 & 18.647 \\
0.5 & 130.65 & 158.24 \\
0.2 & 134.02 & 5713.1 \\
\hline
\end{tabular}

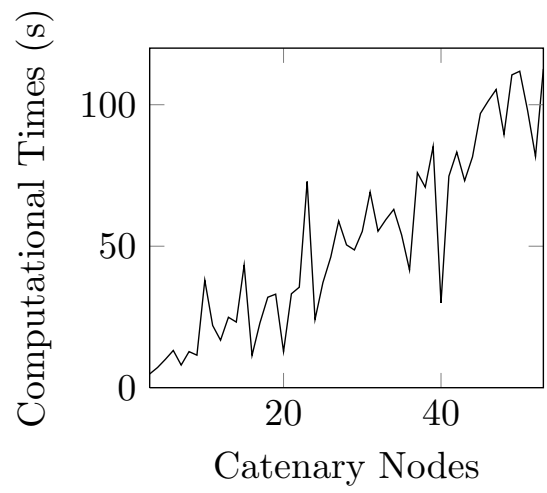

(a) Comparison of computation times for $50 \mathrm{~km}$ catenary, one train, and 3 to 53 catenary nodes.

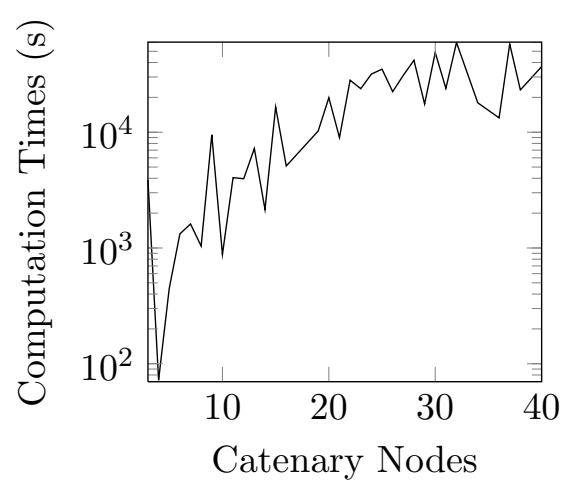

(b) Comparison of computation times for $150 \mathrm{~km}$ catenary, one train, 3 to 40 catenary nodes.

Figure 12: Computational Times in s for various number of catenary nodes.

\subsection{Solver choice impacts}

In the numerical results presented in this paper, the solver SBB (GAMS, 2011) has been used. SBB seems to be the solver that works best with the SOS2 variables. SBB seems on the other hand to very easily find local optima far from the desired solution, so lots of helping constraints (like (44)-(56p) need to be added to obtain technically reasonable solutions.

The solver BONMIN (GAMS, 2011) has been tried out as an alternative, resulting in the same solutions as SBB, but with significantly longer computation time. What for example took about 15 seconds in SBB took about 20 minutes in BONMIN. That minor test was made on a system with merely a 


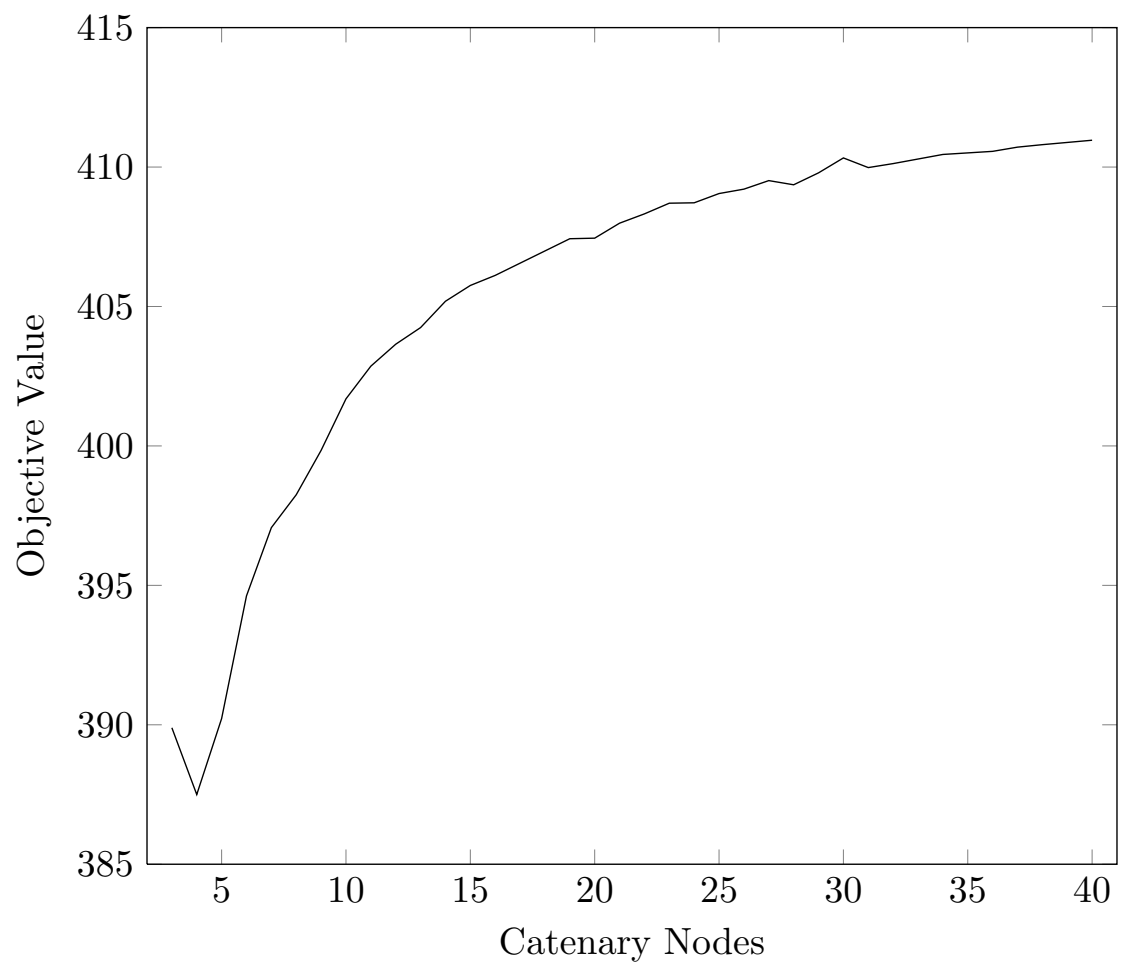

Figure 13: Convergence for the $150 \mathrm{~km}$ case study and increasing numbers of catenary nodes

$20 \mathrm{~km}$ catenary. That test did however include the helping constraints that possibly BONMIN would be fine without.

\subsection{Alternative Objective Functions}

Some other objectives were tried out that logically would also aim at minimizing train traveling times.

Experiments have been done with minimizing negative velocities (e.g. mathematically to

$$
\min z
$$

where

$$
z=\sum_{t, \delta}-v_{t, \delta}
$$


which physically is to maximize the summed train speed over all trains and all time steps), the summed squares of the tractive and the braking forces, and the sums of the driving and braking binary indicator variables. These experiments resulted in shorter computation times, and slightly longer train traveling times. What worse is, they also resulted in edgy (c.f. the example in Figure 14) tractive force curves indicating unrealistic and undesired solutions.

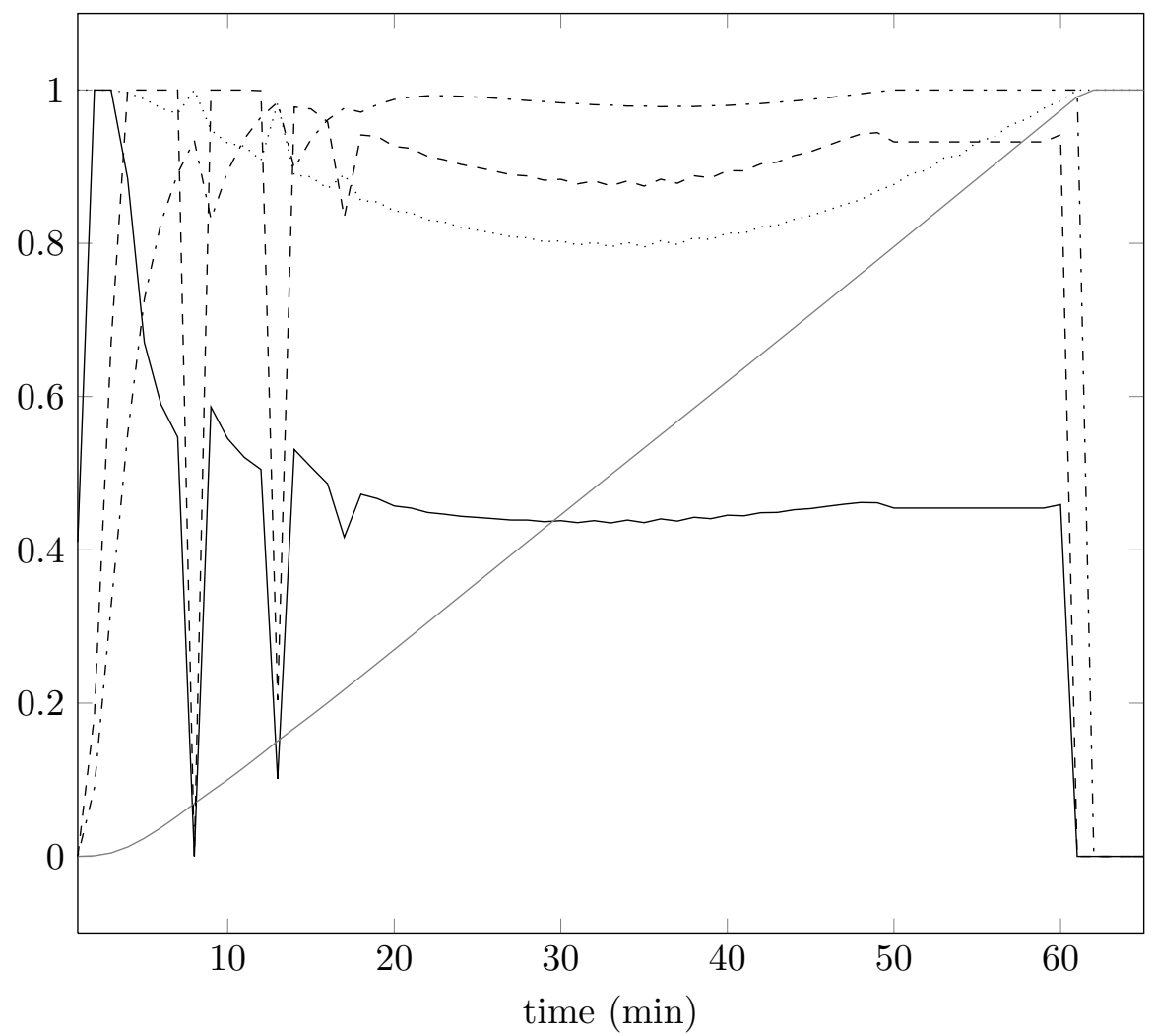

Figure 14: Electrical and mechanical properties of the train plotted against time. All the curves are normalized. Their descriptions and their normalization factors are stated in Table 4. In contrast to Figure 2, where objective function (74) is used, the objective function resulting in the plots of this figure is 80 .

Figure 14 describes the results for an objective function minimizing the 
number of time steps that the train is either driving or braking, i.e.

$$
z=\sum_{t, \delta}\left(d_{t, \delta}+b_{t, \delta}\right)
$$

which can be compared with (74). Comparing Figure 14 with Figure 2 the observant reader sees that the time in traffic using objective 80 results in 61 minutes in traffic, whereas the objective (74) results in 60 minutes in traffic. The alternative objective does not only result in edgier curves, it also results in an inferior result.

\section{Conclusions and Summary of Results}

The main contributions of this paper is the introduction of a new way of modeling moving loads in electric power systems. The model has a fixed number of stationary power system nodes, and is formulated as one single optimization problem, which

- ... enables combined studies of optimal driving strategies and power system operation,

- ... offers a surveyable modeling framework contained in one objective and a set of constraints, and which

- ... makes the model migratable to different hardware and software environments, e.g. to supercomputers. When the entire train movement and power supply problems are congregated into one problem, the communication between different parts of the problem can be unconsidered.

The main conclusion to be drawn from the studies made are that the problem becomes much more complicated to solve when power system matters. It has been shown that a $5 \mathrm{~km}$ nodal distance is enough when minimizing train running time with respect to voltage drops. The computation times are very sensitive to time step sizes due to extreme memory consumption for high temporal resolution.

A useful way of modeling a moving load RPSS as a fixed-node power system has been presented and proven to be useful. The method is able to produce exact and trustworthy results. With this type of moving-load power system model, the electrical nodes are fixed to their number, their numbering, 
and their locations - there is no update of the admittance matrix during the computation.

For the application of electric railways, the computational times increase significantly when voltage drops start to affect train traffic performance due to problem complexity. That is when the mechanical and electrical parts of the system are interacting. Shorter discretization time steps also result in increased computational times, in this case due to problem size.

The temporal part of the model has uniform time steps. The model is on the other hand general in the catenary nodes discretization allowing nonuniform sampling.

Electrical accuracy is increased by denser located catenary nodes, and mechanical accuracy is increased by smaller time steps. Accuracy has a price - computer time.

One can also conclude that, due to the computational times on a normal $\mathrm{PC}$ workstation, if optimal driver strategies or charge/discharge strategies or similar time-dependent issues are not of interest in a particular study, a classical simulation software or a revised model might be to prefer.

\section{Discussions and Future Work}

The proposed solution imposes a large number of nodes for more exact electrical studies. From the perspective of computational burdens, this overweighs the alternative with rearranged nodal matrices for each time step and nodes only representing moving loads and fixed power system nodes (Talukdar and Koo, 1977). So for studies not aiming at optimization over time, this model is not to prefer computationally. It does however offer a convenient, portable, and comprehensible modeling framework, and therefore a revised model not optimizing over time is worth the consider of development. If choosing not to optimize over time, and implicitly decoupling the time steps, the computations will be sped up.

Keeping the SOS2 formulation for easy and neat modeling and bookkeeping, the model could be reformulated such that each time step is optimized for consecutively. Then, in addition, there will not be any SOS2 variable in the optimization anymore, the linear weighting will instead be managed by solving a small system of equations separately for each time step.

Since the model focuses on the power system, the mechanical modeling has been simplified. For proper models of train-rail interaction, c.f. (Lukaszewicz, 
2001). Less simplified mechanical models including proper models of the power system are presented in (Abrahamsson, 2008; Boullanger, 2009).

\subsection{Node density and accuracy}

\subsubsection{For strong grids}

As long as the studies only focus on possible train tractive performances, there is no accuracy increase to have many electrical nodes along the catenary when the grid is comparatively strong in relation to the loads. When studying weaker or heavier loaded grids node densities matter. In studies where also energy consumption, peak loads and such are studied, one can expect the accuracy to be increased somewhat, for increased number of nodes regardless of power system strength.

\subsubsection{Generally}

A nonuniform spatial sampling, where sampling is made denser in the middle of the catenary section, where voltage drops are more likely, could be expected to create a good tradeoff between accuracy and computational times.

\subsection{Applications to converter control laws}

Within the existing modeling framework, optimal operation and control laws of converters (Abrahamsson et al., 2012) could be added. Not only converters feeding the railway from the public grid or from a railway transmission line could be optimally operated. That can also be done for converters regulating the charge/discharge of energy storage, or for the on-train converters. It is e.g. in (Ciccarelli et al., 2012) suggested to charge the super-capacitor when braking, discharge it when accelerating, and do nothing when cruising. It can be expected to be more optimal to charge also when cruising if the catenary voltage is at acceptable levels.

\subsection{Solver Choice and Modeling}

It could be of interest rewriting the SOS2 variable formulation as an equivalent system of binary variables in order to see if the model can be solved well by other solvers not as adapted to the SOS2 variables as the SBB solver seems to be. It can be expected that solvers like BONMIN are more accurate, but also demands too extensive computational resources that a personal computer is not sufficient. 
In generalized future models, for example with changing speed-limits, the trains must be allowed to be braking and driving alternately so the equation (44) may have to be altered or taken out from the model. Moreover, this would make the variable $h_{t, \delta}$ obsolete and taken out of the model. That in turn would also make equations (53)-(55) obsolete, resulting in a more general model.

Note that the purpose with this paper is not to present a completely realistic RPSS model, but to present a new concept of modeling moving power system loads that may appear and disappear within the time-frame studied. Now that the model of this paper is presented and justified, further improvements are to come.

Integer variables potentially slow down optimization problems significantly, especially problems with nonlinear constraints. Binary variables in vector form that only change value once could be reformulated as SOS1 variables.

SOS1 variables have been tried out during the model development process to model trains that have started to brake and trains that have finally stopped. The computational times were not significantly reduced then. The potential in using SOS1 variables instead of binaries could however still be further studied, also to indicate that a train has started from standstill position.

Typical for this problem is that many different physical units are used, scaled and unscaled. Therefore, it is expected that the numerical performance would be improved by scaling (Pierre, 1987; Gajulapalli and Lasdon, 2006).

Track topography is normally stored in databases where the inclination is given in per mille, and the radius curvature is given in $\mathrm{m}$, for different positions. It is assumed the the inclination or curvature is kept constant until it is updated in the database. The position-dependent grade and curvature parts of the running resistances could with favor be modeled using SOS2 variables.

In this paper, the focus was set on the new type of modeling the train positions in the electric grid, and on the opportunities with the model. Future more physically realistic models should sonsider the real adhesive train masses, and that train accelerations is computed differently depending on if the train is braking, coasting, and motoring/regenerating. In real-life, only locomotives without carriages and some types of multiple-unit-trains have adhesive masses and total train masses the same. More detailed mechanical models could also consider rotational inertia in the wheel sets and in the 
locomotive. When adhesive masses are considered, co can also the adhesive forces be - another limiting factor on possible maximal acceleration.

Abrahamsson, L., 2008. Railway Power Supply Models and Methods for Long-term Investment Analysis. Technical Report. Royal Institute of Technology (KTH), Stockholm, Sweden. Licentiate Thesis.

Abrahamsson, L., Kjellqvist, T., Östlund, S., 2012. HVDC Feeder Solution for Electric Railways. IET Power Electronics. In Press.

Abrahamsson, L., Söder, L., 2012. An SOS2-based moving trains, fixed nodes, railway power system simulator, in: Presented at COMPRAIL 2012. To appear in the 2014 preceedings. Available online at: http: //www.algonet.se/ asch/Comprail12/comprail12.pdf.

Arboleya, P., Diaz, G., Coto, M., 2012. Unified ac/dc power flow for traction systems: A new concept. IEEE Journal of Vehicular Technology 61, 24212430. Accepted for publication.

Barrero, R., Tackoen, X., Mierlo, J.V., 2008. Quasi-static simulation method for evaluation of energy consumption in hybrid light rail vehicles, in: Vehicle Power and Propulsion Conference, 2008. VPPC '08. IEEE, pp. 1-7.

Boullanger, B., 2009. Modeling and simulation of future railways. Master's thesis. Royal Institute of Technology (KTH).

Ciccarelli, F., Iannuzzi, D., Tricoli, P., 2012. Control of metro-trains equipped with onboard supercapacitors for energy saving and reduction of power peak demand. Transportation Research Part C: Emerging Technologies $24,36-49$.

Gajulapalli, R.S., Lasdon, L.S., 2006. Scaling Sparse Constrained Nonlinear Problems for Iterative Solvers. Technical Report. Indian Institute of Management, Ahmedabad, India. http://www.iimahd.ernet.in/assets/ snippets/workingpaperpdf/2006-08-06gsravindra.pdf.

GAMS, 2008. Welcome to the GAMS Home Page! http://www.gams.com.

GAMS, 2011. Solver descriptions. http://www.gams.com/solvers/ solvers.htm. 
Hill, R.J., 1994. Electric railway traction, Part 3 Traction power supplies. Power Engineering Journal 8, 275-286.

Iannuzzi, D., Ciccarelli, F., Lauria, D., 2012. Stationary ultracapacitors storage device for improving energy saving and voltage profile of light transportation networks. Transportation Research Part C: Emerging Technologies $21,321-337$.

Iannuzzi, D., Lauria, D., Tricoli, P., 2011. Optimal design of stationary supercapacitors storage devices for light electrical transportation systems. Optimization and Engineering DOI: 10.1007/s11081-011-9160-4.

Iannuzzi, D., Tricoli, P., 2012. Speed-based state-of-charge tracking control for metro trains with onboard supercapacitors. Power Electronics, IEEE Transactions on 27, 2129-2140.

López-López, A.J., Pecharromán, R.R., García-Matos, J.A., FernándezCardador, A., Cucala, A.P., 2012. Optimal deployment of energy storage systems in a DC-electrified railway system, in: Computers in Railways XIII, WIT Press. pp. 603-614.

Lukaszewicz, P., 2001. Energy Consumption and Running Time for Trains. Ph.D. thesis. Division of Railway Technology, KTH, Stockholm, Sweden.

McCarl, B.A., Meeraus, A., van der Eijk, P., Bussieck, M., Dirkse, S., Steacy, P., Nelissen, F., 2012. McCarl GAMS User Guide, Version 23.8.

Pierre, D., 1987. An optimal scaling method. Systems, Man and Cybernetics, IEEE Transactions on 17, $2-6$.

Shiokawa, K., Tagaki, R., 2012. Numerical optimisation of the charge/discharge characteristics of wayside energy storage systems by the embedded simulation technique using the railway power netwrok simulator RTSS, in: Computers in Railways XIII, WIT Press. pp. 583-592.

Talukdar, S.N., Koo, R.L., 1977. The analysis of electrified ground transportation networks. IEEE Transactions on Power Apparatus and Systems 96, 240-247. 